\title{
Chemical abundances and radial velocities in the extremely metal-poor galaxy DDO 68
}

\author{
F. Annibali, ${ }^{1 \star}$ V. La Torre,${ }^{2}$ M. Tosi,${ }^{1}$ C. Nipoti, ${ }^{2}$ F. Cusano, ${ }^{1}$ A. Aloisi,${ }^{3}$ \\ M. Bellazzini, ${ }^{1}$ L. Ciotti, ${ }^{2}$ A. Marchetti, ${ }^{4}$ M. Mignoli, ${ }^{1}$ D. Romano, ${ }^{1}$ E. Sacchi. ${ }^{3}$ \\ ${ }^{1}$ INAF-Osservatorio di Astrofisica e Scienza dello Spazio, Via Gobetti 93/3, I-40129 Bologna, Italy \\ ${ }^{2}$ Dipartimento di Fisica e Astronomia, Bologna University, Via Gobetti 93/2, I-40129, Bologna, Italy \\ ${ }^{3}$ Space Telescope Science Institute, 3700 San Martin Drive, Baltimore, MD 21218 \\ ${ }^{4}$ INAF-Istituto di Astrofisica Spaziale e Fisica Cosmica, Via Bassini 15, I-20133 Milano, Italy
}

Accepted XXX. Received YYY; in original form ZZZ

\begin{abstract}
We present chemical abundances and radial velocities of six $\mathrm{H}$ II regions in the extremely metal-poor star-forming dwarf galaxy DDO 68. They are derived from deep spectra in the wavelength range 3500 - 10,000 $\AA$, acquired with the Multi Object Double Spectrograph (MODS) at the Large Binocular Telescope (LBT). In the three regions where the $[\mathrm{O}$ III $] \lambda 4363 \AA$ line was detected, we inferred the abundance of $\mathrm{He}, \mathrm{N}$, $\mathrm{O}, \mathrm{Ne}, \mathrm{Ar}$, and $\mathrm{S}$ through the "direct" method. We also derived the oxygen abundances of all the six regions adopting indirect method calibrations. We confirm that DDO 68 is an extremely metal-poor galaxy, and a strong outlier in the luminosity - metallicity relation defined by star-forming galaxies. With the direct-method we find indeed an oxygen abundance of $12+\log (\mathrm{O} / \mathrm{H})=7.14 \pm 0.07$ in the northernmost region of the galaxy and, although with large uncertainties, an even lower $12+\log (\mathrm{O} / \mathrm{H})=6.96 \pm 0.09$ in the "tail". This is, at face value, the most metal-poor direct abundance detection of any galaxy known. We derive a radial oxygen gradient of $-0.06 \pm 0.03 \mathrm{dex} / \mathrm{kpc}$ (or -0.30 dex $R_{25}^{-1}$ ) with the direct method, and a steeper gradient of $-0.12 \pm 0.03 \mathrm{dex} / \mathrm{kpc}$ (or $-0.59 \operatorname{dex} R_{25}^{-1}$ ) from the indirect method. For the $\alpha$-element to oxygen ratios we obtain values in agreement with those found in other metal-poor star-forming dwarfs. For nitrogen, instead, we infer much higher values, leading to $\log (\mathrm{N} / \mathrm{O}) \sim-1.4$, at variance with the suggested existence of a tight plateau at -1.6 in extremely metal poor dwarfs. The derived helium mass fraction ranges from $Y=0.240 \pm 0.005$ to $Y=0.25 \pm 0.02$, compatible with standard big bang nucleosynthesis. Finally, we measured $\mathrm{H}$ II region radial velocities in the range $479-522 \mathrm{~km} / \mathrm{s}$ from the tail to the head of the "comet", consistent with the rotation derived in the HI.
\end{abstract}

Key words: galaxies: abundances - galaxies: dwarf - galaxies: individual (DDO 68)galaxies: starburst - ISM: H II regions

\section{INTRODUCTION}

DDO 68 (UGC 5340, SDSS J0956+2849) is a star-forming dwarf galaxy of special interest in the framework of the $\Lambda$ Cold Dark Matter $(\Lambda \mathrm{CDM})$ scenario for galaxy formation, because it is one of the first observed systems confirming the prediction that dark matter haloes host substructures down to the resolution limit of the simulations (Diemand et al. 2008; Wheeler et al. 2015), i.e., down to the smallest mass

^ E-mail: francesca.annibali@inaf.it scales. In fact, in spite of being located in the huge LynxCancer void (Pustilnik \& Tepliakva 2011) and of having formed a total mass of stars of only $10^{8} M_{\odot}$ (Sacchi et al. 2016), DDO 68 clearly suffers the presence of at least three smaller companions, thus appearing as a flea with smaller fleas that on him prey ${ }^{1}$ (Annibali et al. 2016).

DDO 68 is at a distance of $\sim 12.7 \mathrm{Mpc}$ from us

\footnotetext{
${ }^{1}$ From Jonathan Swift's On Poetry: a Rhapsody: So, naturalists observe, a flea/ has smaller fleas that on him prey;/ and these have smaller still to bite 'em/ and so proceed ad infinitum.
} 
(Cannon et al. 2014; Sacchi et al. 2016; Makarov et al. 2017). With an oxygen abundance of $12+\log (\mathrm{O} / \mathrm{H}) \sim 7.14$ measured in a few $\mathrm{H}$ II regions at its northern edge (Pustilnik et al. 2005, 2007; Izotov \& Thuan 2007, 2009), about $1 / 40$ of solar (in Caffau et al. 2008, scale), it is an extremely metal-poor (XMP) dwarf, much metalpoorer than typical dwarfs of similar mass (Pustilnik et al. 2005). As a consequence, DDO 68 strongly deviates from the mean luminosity-metallicity relation defined by starforming galaxies in the Local Universe (Lequeux et al. 1979; Skillman et al. 1989; Berg et al. 2012).

Because of its very distorted morphology, DDO 68 was suggested to be affected by galaxy interaction already years ago (Ekta, Chengalur \& Pustilnik 2008; Cannon et al. 2014; Tikhonov, Galazutdinova, \& Lebedev 2014). Ekta et al. noticed distortions in the $\mathrm{H}$ I distribution and interpreted the observed features in terms of a late-stage merger of two gasrich progenitors. Tikhonov et al. proposed that the portion of the galaxy that appears as a cometary tail is actually a disrupted satellite currently being cannibalized by DDO 68 , while Cannon et al. identified a possible $\mathrm{H}$ I satellite, with $M_{\mathrm{HI}} \simeq 3 \times 10^{7} M_{\odot}$, at a projected distance of $\simeq 40 \mathrm{kpc}$ from the main body. Annibali et al. (2016), combining Hubble Space Telescope (HST) and Large Binocular Telescope (LBT) photometry, showed that, in addition to the known cometary tail, there is a faint, small stellar stream falling on the main body of DDO 68 and an arc possibly resulting from tidal interactions. They found that the morphologies of tail, stream, arc and main body are well reproduced by N-body simulations of the interaction among three distinct bodies, the main body and two smaller ones with masses $1 / 10$ and $1 / 150$ the mass of the main body.

The faint stream is populated mainly by red giant branch (RGB) stars (Annibali et al. 2016), while the cometary tail hosts stars of all ages (Tikhonov, Galazutdinova, \& Lebedev 2014; Sacchi et al. 2016; Makarov et al. 2017) and is particularly rich in H il regions. From deep and accurate Colour-Magnitude diagrams (CMDs) obtained from HST photometry, Sacchi et al. (2016) inferred a robust spatially resolved star formation history (SFH) of the various regions of DDO 68, finding that everywhere stars were already forming at the oldest lookback time reached by the photometry, i.e. at least 2-3 Gyr ago, corresponding to the minimum age of the measured RGB stars. The measured RGB stars may actually be up to 13 Gyr old, but the age-metallicity degeneracy affecting the RGB prevents one to precisely age-date these stars, since independent spectroscopic estimates of their metallicity are not available. According to Sacchi et al., DDO 68 is likely to have formed stars at a low rate during its whole life, with a significant enhancement a few hundred million years ago, a peak activity of $\sim 4.6 \times 10^{-2}$ $\mathrm{M}_{\odot} \mathrm{yr}^{-1} \mathrm{kpc}^{-2}$ between 20 and $60 \mathrm{Myr}$ ago, and a current much lower rate.

If the galaxy has formed stars for a Hubble time, although at a relatively low rate, how did it manage to remain so metal-poor? Is it because it has recently accreted significant amounts of very low-metallicity gas that diluted its medium, or because it got rid through differential galactic winds of the elements produced by its stars? Chemical evolution models of late-type dwarfs have often invoked both effects to reproduce the observed low chemical abun- dances of star-forming dwarfs (see e.g., Matteucci \& Tosi 1985; Pilyugin 1992; Marconi et al. 1994; Romano et al. 2006; Recchi et al. 2008; Romano \& Starkerburg 2013), suggesting that the outflows triggered by supernova explosions in these shallow potential well systems may be more efficient in ejecting some of the heavy elements (like the $\alpha$ elements and iron) than other chemical species such as $\mathrm{H}$ and He. Indeed, hydrodynamical simulations of late-type dwarfs (e.g., D'Ercole \& Brighenti 1999; Mac Low \& Ferrara 1999; Scannapieco \& Brüggen 2010) clearly show that the outflows that blow out of these galaxies drive substantial amounts of the metals produced in the starburst into the intergalactic medium. Other authors have instead considered the infall of primordial gas sufficient (see, e.g. Gavilàn et al. 2013) to reproduce the observed metallicities. It has also been suggested (e.g. Legrand et al. 2000) that a continuous, very low star formation regime could account for the observed abundances in some extremely metal poor dwarf galaxies.

The combination of a detailed SFH with chemical abundances derived from accurate spectroscopy and with constraints on the dynamics of its various constituents is a key ingredient to reconstruct a coherent and complete picture of how DDO 68 (and, more in general, XMPs) formed and evolved. To this aim, here we exploit the Multi Object Double Spectrograph (MODS) mounted on the LBT to study DDO 68's H in regions.

In this paper, Section 2 describes the observations and data reduction, and Section 3 presents the procedure for the derivation of the reddening-corrected emission line fluxes. The results of temperatures, densities, chemical abundances are described in Section 4, while the derived abundances, abundance ratios and abundance spatial distributions are described in Section 5. Radial velocities are presented and discussed in Section 6. The overall results are discussed and summarised in Section 7.

\section{OBSERVATIONS AND DATA REDUCTION}

Our observations (LBT program 2016-2017-50, PI Annibali) were performed on February 21, 2017, with MODS1 and MODS2 mounted on LBT. The LBT, located at Mt. Graham (Arizona), is composed of two identical $8.4 \mathrm{~m}$ telescopes that can work simultaneously in binocular mode, and it is larger than all telescopes used for previous optical spectroscopic studies of DDO 68. When used in dichroic mode, the MODS spectrographs provide high quality spectra in the $\sim 3400-5500 \AA$ and $\sim 5700-9800 \AA$ ranges for the blue and red channels, respectively. The $6^{\prime} \times 6^{\prime}$ field of view of MODS is perfectly suited to cover the whole DDO 68 system and its surroundings. The target $\mathrm{H}$ II regions were selected from our HST (GO program 11578; PI: Aloisi) V, I, and H $\alpha$ images. In those images, $\mathrm{H}$ II regions are resolved and appear as regions of diffuse $\mathrm{H} \alpha$ and V (i.e. [OIII] $\lambda \lambda 4959,5007)$ emission. In order to look for differences (or lack thereof) in the abundances of the different structures, we selected H II regions covering all the main structural zones of DDO 68, and targeted six of them for our observations. According to $\mathrm{H} \alpha$ images from our HST program and from Pustilnik et al. (2005), no emission line region is found along the faint stream discovered by Annibali et al. (2016). In addition, we targeted a candidate star cluster (CL) and two potential companions of DDO 68 


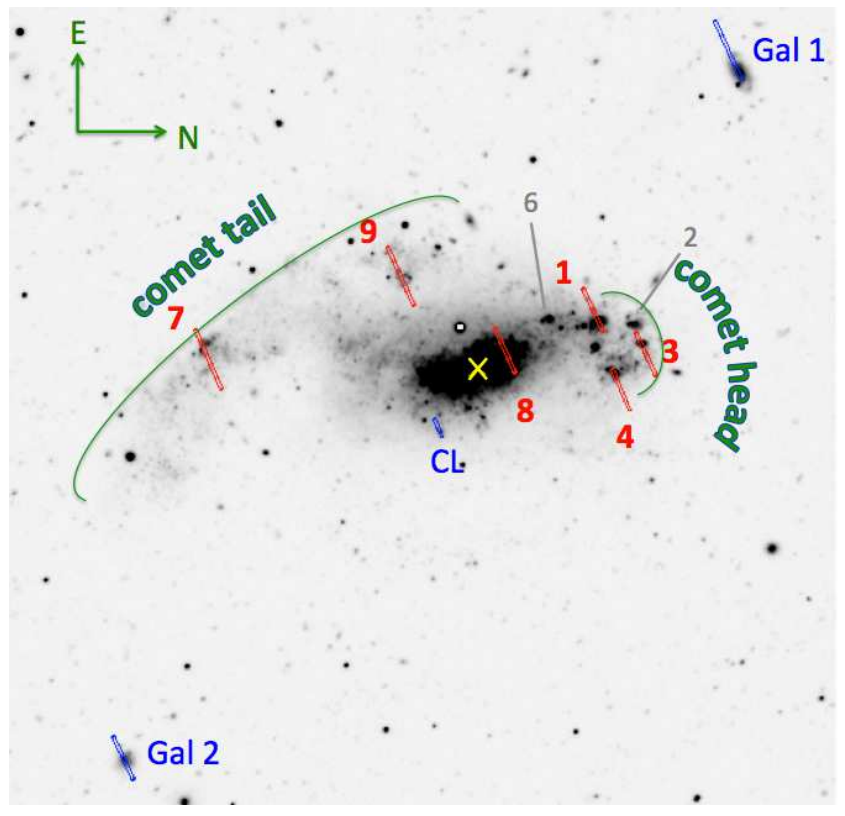

Figure 1. LBT $g$ image of DDO 68, showing the position of our 9 MODS slits. Six slits $(1,3,4,7,8$ and 9) were centred on $\mathrm{H}$ II regions in DDO 68, two slits (Gal 1 and Gal 2) on two candidate galaxy companions, and one slit (dubbed CL) on a candidate star cluster in DDO 68. For completeness, we also indicate (in grey) Reg. 2 and Reg. 6 identified by Pustilnik et al. (2005) and not observed in our study because of slit positioning constraints. The head and the tail of the "comet" are indicated on the image. The yellow cross corresponds to the centre of the galaxy identified from isophotal contours derived from the LBT $g$ image.

detected in our LBT images (dubbed Gal 1 and Gal 2). An LBT image of DDO 68 with overimposed the positions of our 9 slits is shown in Figure 1.

Four of our target H II regions were already observed by Pustilnik et al. (2005) and Izotov \& Thuan (2009); for them we adopt the names assigned by those authors, i.e. regions 1, 3, 4, and 7 (see Fig. 1.) Regions 8 and 9 are instead newly observed H II regions. One H II region (Reg. 8) is close to the centre of the main body, two (Reg. 7 and Reg. 9) lie along the cometary tail, and three (Reg. 1, Reg. 3 and Reg. 4) in the northern ring-like structure that some consider the head of the comet (Makarov et al. 2017). Reg. 3 also includes the Luminous Blue Variable (LBV) studied by Izotov \& Thuan (2009) and Pustilnik et al. (2016)

Observations and data reduction have been designed following the same strategy devised by Annibali et al. (2017) for the star-forming irregular galaxy NGC 4449. We have crafted the masks using our HST images, and adopting a slit width of $1^{\prime \prime}$; for the H II regions, we adopted slit lengths between 15 " and 20", sufficient to include also a portion of the background without significant nebular emission. We designed two different masks, implemented in two separate runs (Run A and Run B) to be able to place the slits on all our 9 target regions. We observed our targets using the blue G400L (3200-5800 ̊) and the red G670L (5000-10000 $\AA$ ) gratings on the blue and red channels in dichroic mode, to cover the spectral range from the $[\mathrm{OII}] \lambda 3727$ nebular line to the [SIII] $\lambda 9530$ line, allowing the derivation of the abun- dances of He, N, O, Ne, S, and Ar. The observations were performed in binocular mode using MODS1 and MODS2 simultaneously. The exposure times were dictated by the need to observe the relatively faint [OIII] $\lambda 4363$ auroral line with a signal/noise of at least 3 , to derive the nebular electronic temperatures. We had 12 exposures in Run A and 10 in Run B of $600 \mathrm{sec}$ each, for a total of less than 4 hours. The seeing varied between $\sim 0.5 "$ and $\sim 0.8 "$, and the airmass from $\sim 1.0$ to $\sim 1.1$. In order to avoid significant effects from differential atmospheric refraction (see e.g. Filippenko 1982), the slits were approximately oriented along the parallactic angle (see Table 1). We used the MODS observing planning tool ${ }^{2}$ to check that our observations were not affected by flux losses due to differential atmospheric refraction indeed.

The data acquired with MODS1 and MODS2 were treated separately, since the two instruments turned out to have different sensitivities. However, to allow for the potential detection of intrinsically faint, key lines (e.g., [O III] 24363), we also produced deep spectra combining together all the MODS1 and MODS2 sub-exposures. Bias and flat-field corrections, and wavelength calibration were executed by the Italian LBT Spectroscopic Reduction Facility at INAF-IASF Milano. They provided the calibrated two-dimensional (2D) spectra for the individual subexposures. The accuracy of the wavelength calibration obtained from the pipeline was checked against prominent sky lines adopting the nominal sky wavelengths tabulated in Hanuschik (2003). Then, the individual sub-exposures were sky-subtracted and combined into final $2 \mathrm{D}$ blue and red frames. The sky subtraction was performed with the background task in $\mathrm{IRAF}^{3}$, typically choosing the windows at the two opposite sides of the central source. The typical size of our H II regions (FWHM $\lesssim 2$ ") is significantly smaller than the adopted slit lengths of 15 "-20", allowing for an adequate removal of the sky background. The one-dimensional (1D) spectra were extracted from the $2 \mathrm{D}$ wavelength-calibrated and sky-subtracted spectra by running the apall task in the twodspec IRAF package. The effective spectral resolution of our spectra, as measured from prominent sky lines in the 1D background-unsubtracted spectra, is $\mathrm{R} \sim 1400$ at $\lambda \sim 5500$ $\AA$ and $\mathrm{R} \sim 1000$ at $\lambda \sim 7200 \AA$.

The blue and red 1D spectra were flux calibrated using the sensitivity curves from the Italian LBT spectroscopic reduction pipeline; the curves were derived using the spectrophotometric standard star GD 71, observed with both MODS1 and MODS2 in dichroic mode with a $5^{\prime \prime}$-width slit on the same night as our targets and at the same airmass. Each MODS1/MODS2 spectrum was calibrated using the appropriate sensitivity functions, while the deep, total combined spectra were calibrated through an average sensitivity curve. After calibration, the MODS1, MODS2, and total combined spectra turned out to typically agree within 5-10\%. The final calibrated MODS1 and MODS2 spectra of our nine targets are shown in Figure 2 and Figures A1 to A 8 in the Appendix. As shown by the spectra, Gal. 1 and

2 www.astronomy.ohio-state.edu/MODS/ObsTools/modsTools/ 3 IRAF is distributed by the National Optical Astronomy Observatory, which is operated by the Association of Universities for Research in Astronomy, Inc., under cooperative agreement with the National Science Foundation. 
Table 1. Journal of Observations.

\begin{tabular}{|c|c|c|c|c|c|c|c|c|c|}
\hline Run & Exp. n. & Instrument & $\begin{array}{c}\text { Date-obs. } \\
\text { [yyyy-mm-dd] }\end{array}$ & $\begin{array}{l}\text { Time-obs. } \\
\text { [hh:mm:ss] }\end{array}$ & $\begin{array}{l}\text { Exp.Time } \\
{[\mathrm{s}]}\end{array}$ & $\begin{array}{l}\text { Seeing } \\
\text { [arcsec] }\end{array}$ & Airmass & $\begin{array}{l}\mathrm{PA} \\
{\left[{ }^{\circ}\right]}\end{array}$ & $\begin{array}{c}\text { PARA } \\
{\left[{ }^{\circ}\right]}\end{array}$ \\
\hline \multirow{10}{*}{ B } & 1 & MODS1 & 2017-02-21 & $05: 22: 48$ & 600. & $0.6 "-0.7 "$ & 1.09 & -65 & -74 \\
\hline & 2 & MODS2 & $2017-02-21$ & $05: 22: 28$ & 600. & $0.6 "-0.7 "$ & 1.09 & -65 & -74 \\
\hline & 3 & MODS1 & $2017-02-21$ & $05: 34: 32$ & 600. & $0.6 "-0.7 "$ & 1.07 & -65 & -73 \\
\hline & 4 & MODS2 & $2017-02-21$ & $05: 34: 16$ & 600. & $0.6 "-0.7 "$ & 1.07 & -65 & -73 \\
\hline & 5 & MODS1 & $2017-02-21$ & $05: 46: 16$ & 600. & $0.6 "-0.7 "$ & 1.06 & -65 & -73 \\
\hline & 6 & MODS2 & $2017-02-21$ & $05: 47: 12$ & 600. & $0.6 "-0.7 "$ & 1.06 & -65 & -73 \\
\hline & 7 & MODS1 & $2017-02-21$ & 05:58:00 & 600. & $0.6 "-0.7 "$ & 1.04 & -65 & -72 \\
\hline & 8 & MODS2 & $2017-02-21$ & $05: 57: 60$ & 600. & $0.5 "-0.6 "$ & 1.04 & -65 & -72 \\
\hline & 9 & MODS1 & 2017-02-21 & 06:09:44 & 600. & $0.5 "-0.6 "$ & 1.03 & -65 & -70 \\
\hline & 10 & MODS2 & 2017-02-21 & $06: 10: 47$ & 600. & $0.5 "-0.6 "$ & 1.03 & -65 & -70 \\
\hline \multirow{12}{*}{ A } & 1 & MODS1 & $2017-02-21$ & 06:38:02 & 600. & $0.5 "-0.6 "$ & 1.01 & -65 & -59 \\
\hline & 2 & MODS2 & $2017-02-21$ & $06: 37: 40$ & 600. & $0.5 "-0.6 "$ & 1.01 & -65 & -59 \\
\hline & 3 & MODS1 & $2017-02-21$ & $06: 49: 47$ & 600. & $0.5 "-0.6 "$ & 1.01 & -65 & -48 \\
\hline & 4 & MODS2 & $2017-02-21$ & $06: 49: 27$ & 600. & $0.5 "-0.6 "$ & 1.01 & -65 & -48 \\
\hline & 5 & MODS1 & 2017-02-21 & 07:01:31 & 600. & $0.5 "-0.6 "$ & 1.00 & -65 & -27 \\
\hline & 6 & MODS2 & $2017-02-21$ & 07:01:15 & 600. & $0.5 "-0.6 "$ & 1.00 & -65 & -27 \\
\hline & 7 & MODS1 & $2017-02-21$ & 07:13:15 & 600. & $0.5 "-0.6 "$ & 1.00 & -65 & 8 \\
\hline & 8 & MODS2 & $2017-02-21$ & 07:13:03 & 600. & $0.5 "-0.6 "$ & 1.00 & -65 & 8 \\
\hline & 9 & MODS1 & $2017-02-21$ & $07: 24: 59$ & 600. & $0.5 "-0.6 "$ & 1.00 & -65 & 37 \\
\hline & 10 & MODS2 & $2017-02-21$ & $07: 25: 50$ & 600. & $0.5 "-0.6 "$ & 1.00 & -65 & 37 \\
\hline & 11 & MODS1 & $2017-02-21$ & $07: 36: 43$ & 600. & $0.8 "$ & 1.01 & -65 & 54 \\
\hline & 12 & MODS2 & $2017-02-21$ & $07: 36: 38$ & 600. & $0.8 "$ & 1.01 & -65 & 54 \\
\hline
\end{tabular}

Col. (1): run of observations; Col. (2): exposure number; Col. (3): instrument (MODS1 or MODS2); Col. (4): date of observations; Col. (5): UTC time of observations; Col. (6): duration of exposure; Col. (7): seeing in arcsec; Col. (8): airmass; Col. (9): slit position angle; Col. (10): parallactic angle.

Gal. 2 turned out to be background emission-line galaxies at redshifts of $\mathrm{z} \sim 0.11$ and $\mathrm{z} \sim 0.0148$, respectively, and CL an early-type galaxy at $\mathrm{z} \sim 0.42$. Since they are not related to DDO 68 , these systems will be no longer discussed in the paper.

\section{EMISSION LINE MEASUREMENT}

Emission line fluxes were measured with the deblend function in the splot IRAF task. We fitted all together lines close in wavelengths (e.g. $\mathrm{H} \beta$, HeI $\lambda 4922$, [O III] $\lambda \lambda 4959,5007$, HeI $\lambda 5015$ ) assuming a Gaussian profile, leaving the centroids and FWHM as free parameters, but imposing the FWHMs to be the same for all fitted lines. The continuum was defined choosing two continuum windows to the left and to the right of the line or line complex, and fitting it with a linear regression. In the case of the $[\mathrm{N}$ II $] \lambda \lambda 6548,84$ lines, blended with the much stronger $\mathrm{H} \alpha$ emission and therefore difficult to measure, we did not leave the centroids as free but allowed a single shift with respect to the nominal zerovelocity line wavelengths. Furthermore, we introduced an additional broad $\mathrm{H} \alpha$ component in the fit of Reg. 1 and 3 to properly reproduce the observed wings. The final emission fluxes were obtained repeating the measurement several times with slightly different continuum choices, and averaging the results. The procedure was applied separately to the MODS1 and the MODS2 spectra. We found that in general MODS2 provided fluxes about 5\% higher than MODS1. The final line fluxes were computed averaging the results from MODS1 and MODS2, and the errors were computed as the standard deviation from the two different measurements. The detection of intrinsically faint lines that are key to the determination of the electronic temperatures (e.g., [O III] $\lambda 4363$, [S III] 26312 , [O II] $\lambda \lambda 7320,30)$ was checked on the $2 \mathrm{D}$ spectra. The $[\mathrm{O}$ III $] \lambda 4363$ line is well detected in Reg. 1 and Reg. 3, is quite faint in Reg. 7, and is not detected in the other regions. For Reg. 7, the measurement of this important feature was performed on the deep total combined spectrum.

Reg. 3 is known to host a LBV, discovered by Pustilnik et al. (2008) from the presence of broad emission components, P-Cygni profiles, and spectral variability. The LBV experienced a very rare event of a giant eruption during the years 2008-2011, and was in a "faint" state during the years 2015-2016 (Pustilnik et al. 2017). During the outburst event, the $\mathrm{H} \beta$ emission of Reg. 3 was observed to overcome that of the [O III] $\lambda 5007$ line, as shown in Fig. 1 of Pustilnik et al. (2008). All these properties are not observed in our data of Reg. 3, which look more simular to the spectrum acquired in 2005 by Pustilnik et al, when the LBV was at its minimum. We conclude therefore that at the epoch of our observations the LBV was, as during the years 20152016 , in a rather quiescent phase.

The derived emission lines for the six H il regions studied in DDO 68 are provided in Table B1 in the Appendix.

Balmer underlying absorption and reddening were derived simultaneously through a $\chi^{2}$ minimization procedure. Based on the measured $\mathrm{H} \delta, \mathrm{H} \gamma, \mathrm{H} \beta$ and $\mathrm{H} \alpha$ fluxes, we constructed the following $\chi^{2}$ from all possible line combinations: 


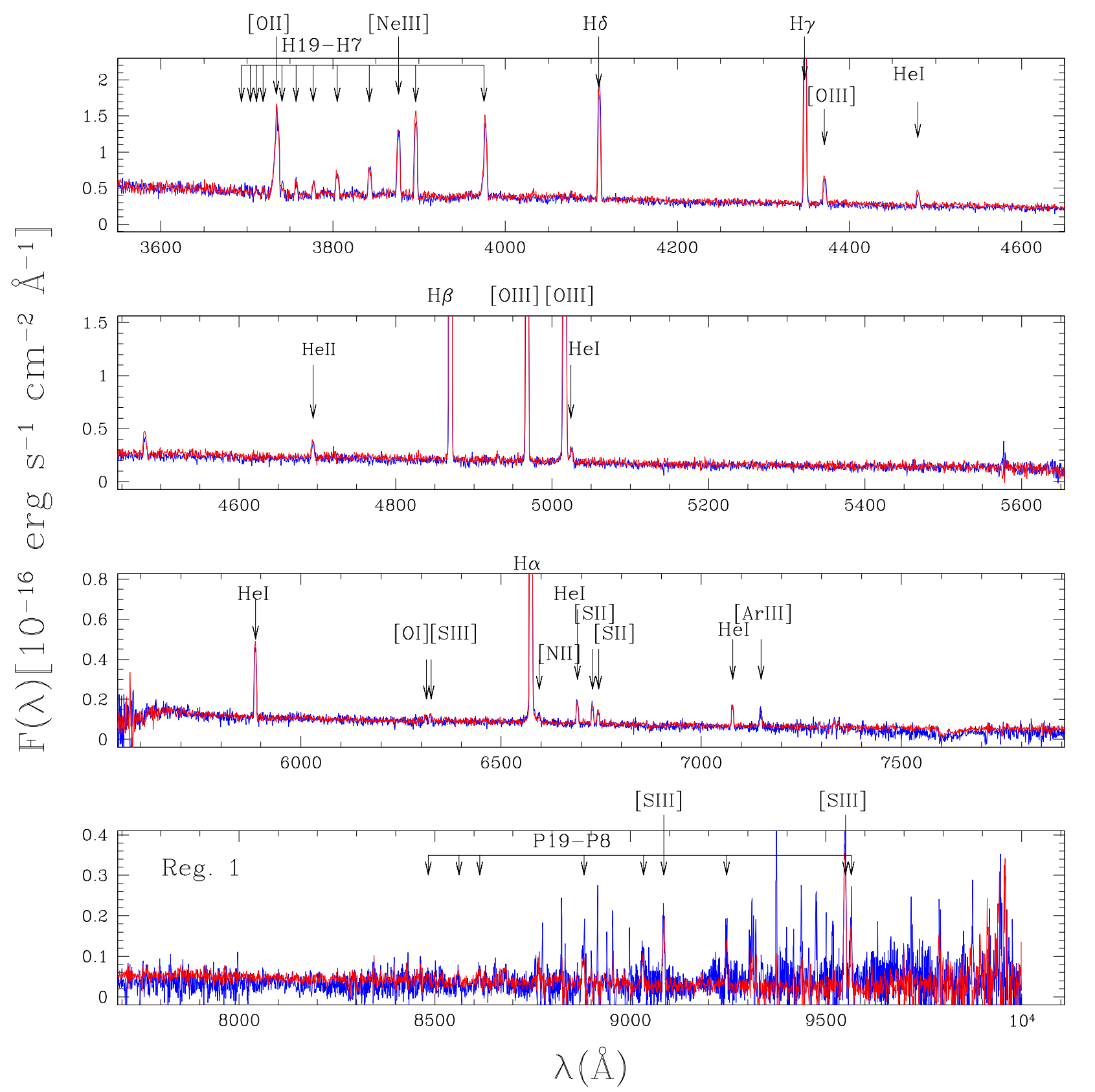

Figure 2. LBT/MODS spectra for Reg. 1 in DDO 68 acquired with MODS1 (blue line) and MODS2 (red line). Indicated are the main identified emission lines. The spectra of the other $\mathrm{H}$ II regions studied in this paper (Reg. 3, 4, 7, 8 and 9), of the two candidate companion galaxies (Gal. 1 and Gal. 2), and of the candidate star cluster (CL) are shown in the Appendix.

$$
\begin{aligned}
\chi^{2}= & \frac{\left(R_{\alpha, \beta}^{\text {corr }}-R_{\alpha, \beta}^{t e o}\right)^{2}}{\sigma_{\alpha, \beta}^{2}}+\frac{\left(R_{\alpha, \gamma}^{\text {corr }}-R_{\alpha, \gamma}^{t e o}\right)^{2}}{\sigma_{\alpha, \gamma}^{2}}+\frac{\left(R_{\alpha, \delta}^{\text {corr }}-R_{\alpha, \delta}^{t e o}\right)^{2}}{\sigma_{\alpha, \delta}^{2}}+ \\
\frac{\left(R_{\beta, \gamma}^{c o r r}-R_{\beta, \gamma}^{t e o}\right)^{2}}{\sigma_{\beta, \gamma}^{2}}+\frac{\left(R_{\beta, \delta}^{c o o r r}-R_{\beta, \delta}^{t e o}\right)^{2}}{\sigma_{\beta, \delta}^{2}}+\frac{\left(R_{\gamma, \delta}^{c o r r}-R_{\gamma, \delta}^{t e o}\right)^{2}}{\sigma_{\gamma, \delta}^{2}}, & =\frac{F_{H \alpha}^{\prime}}{F_{H \beta}^{\prime}} \times 10^{0.4}=\frac{F_{H \alpha}^{\prime}\left(\frac{A_{H \alpha}}{F_{V}^{\prime}}-\frac{A_{H \beta}}{A_{V}}\right) \times R_{V} \times E(B-V)}{0.4\left(A_{H \alpha}-A_{H \beta}\right)}
\end{aligned}
$$

where, for instance, $R_{\alpha, \beta}^{\text {corr }}$ is the reddening-corrected $\mathrm{H} \alpha$ to $\mathrm{H} \beta$ ratio: where $A_{H \alpha} / A_{V}$ and $A_{H \beta} / A_{V}$ are taken from the Cardelli et al. (1989) extinction law with $R_{V}=3.05, E(B-V)$ is the unknown reddening, and $F_{H \alpha}^{\prime}$ and $F_{H \beta}^{\prime}$ are the emis- 
sion fluxes corrected for underlying Balmer absorption according to the formula:

$$
F_{H \alpha, \beta}^{\prime}=F_{H \alpha, \beta}+E W_{a b s} \times C_{H \alpha, \beta} .
$$

In Eq. 3, $F_{H \beta}$ and $F_{H \alpha}$ are the emission fluxes measured on the spectra, $C_{H \beta}$ and $C_{H \alpha}$ are the local continua, and $E W_{a b s}$ is the underlying unknown Balmer absorption in equivalent width assumed to be the same for $\mathrm{H} \delta, \mathrm{H} \gamma, \mathrm{H} \beta$, and $\mathrm{H} \alpha$. This is a reasonable assumption since simple stellar populations models (e.g. Leitherer et al. 2014, hereafter SB99) show that the equivalent width ratios of these absorption lines are close to unity. As for the theoretical Balmer line ratios, we adopted values of $R_{\alpha, \beta}^{t e o}=2.79, R_{\alpha, \gamma}^{t e o}=5.90$, $R_{\alpha, \delta}^{\text {teo }}=10.60, R_{\beta, \gamma}^{\text {teo }}=2.12, R_{\beta, \delta}^{\text {teo }}=3.80, R_{\beta, \delta}^{\text {teo }}=1.80$ assuming case B recombination with $T_{e}=20,000 \mathrm{~K}$ and $n_{e}=100 \mathrm{~cm}^{-3}$ (Osterbrock 1989; Storey \& Hummer 1995), typical of verylow metallicity $\mathrm{H}$ II regions. Finally, the $\sigma$ uncertainties in Eq. (1) are obtained by propagating the flux errors on the emission line ratios.

The $\chi^{2}$ minimization provides reddening values in the range $\mathrm{E}(\mathrm{B}-\mathrm{V}) \sim 0.07-0.24 \mathrm{mag}$ and absorption corrections below EW 1.5 ^ for our six H II regions in DDO 68, consistent with previous works (e.g. Pustilnik et al. 2005). As for Helium lines, the absorption correction was directly estimated from SB99 simple stellar population models with $\mathrm{Z}=0.001$ (the lowest metallicity available for the high-resolution spectra products) and age $<10 \mathrm{Myr}$, resulting into $\mathrm{EW}(\mathrm{He} \mathrm{I} \lambda 4471) \sim 0.5 \AA$, $\mathrm{EW}(\mathrm{He} \mathrm{I} \lambda 5876) \sim 0.5 \AA$, and $\mathrm{EW}($ He I 26678$) \sim 0.3 \AA$. The assumption of a young SSP for the underlying continuum is a reasonable choice since, although DDO 68 experienced a quite continuous SFH from several Gyrs ago until present (Sacchi et al. 2016), the integrated stellar light within its H II regions is dominated by young massive stars. The derived $\mathrm{E}(\mathrm{B}-V)$ values and the final emission-line fluxes corrected for reddening and underlying absorption are provided in Table 2 for our six $\mathrm{H}$ II regions in DDO 68.

\section{TEMPERATURES, DENSITIES AND CHEMICAL ABUNDANCES}

Temperatures, densities, and chemical abundances were obtained following the procedure described in Annibali et al. (2017), using the getCrossTemDen and getIonAbundance tasks of the PyNeb code (Luridiana et al. 2015). The getCrossTemDen task simultaneously derives electron densities $\left(n_{e}\right)$ and temperatures $\left(T_{e}\right)$ through an iterative process assuming a density-sensitive and a temperaturesensitive diagnostic line ratio. We used the densitysensitive $\left[\mathrm{S}\right.$ II] $\lambda 6716 / \lambda 6731$ ratio to derive $n_{e}$ and, when possible, three sets of temperature-sensitive line ratios $([\mathrm{O}$ III $] \lambda 4363 / \lambda 4959+\lambda 5007$, [S III $] \lambda 6312 / \lambda 9069$, and $[\mathrm{O}$ II $] \lambda 7320+\lambda 7330 / \lambda \lambda 3726,29)$ to derive the temperature of the $\mathrm{O}^{++}, \mathrm{S}^{++}$, and $\mathrm{O}^{+}$ions, respectively. Table 3 summarises the results obtained for our Hin regions in DDO 68. The derived densities are typically low, with $n_{e} \lesssim 100 \mathrm{~cm}^{-3}$, in agreement with the results found for other star-forming dwarf galaxies (see, e.g., Annibali et al. $2015,2017)$. The $O^{++}$temperature could only be determined for Reg. 1, Reg. 3, and Reg. 7. We found high $\mathrm{T}_{e}\left(O^{++}\right)$ in the range $17,000-19,000 \mathrm{~K}$, in agreement with the results of Pustilnik et al. (2005) and Izotov \& Thuan (2009). The $S^{++}$temperature could only be obtained for Reg. 1, due to the faintness of the [S III] $\lambda 6312$ line in the other regions; the derived value of $\sim 19,000 \mathrm{~K}$ is in very good agreement with the theoretical relation between $T_{e}\left(O^{++}\right)$and $T_{e}\left(S^{++}\right)$proposed by Garnett (1992) (see below). On the other hand, the $T_{e}\left(O^{+}\right)$obtained for Reg. 1 (and for Reg. 4) strongly deviates from the theoretical relations, a problem that was already found and discussed by previous authors (e.g. Kennicutt et al. 2003; Bresolin et al. 2009a; Berg et al. 2015) and that warns against the use of $O^{+}$temperatures directly derived from the [O II] $\lambda \lambda 7320,30 / \lambda \lambda 3726,29$ ratio.

Chemical abundances were derived for Reg. 1, Reg. 3, and Reg. 7 assuming a three-zone model for the electron temperature structure. The $T_{e}\left(\mathrm{O}^{++}\right)$temperature was adopted for the highest-ionization zone $\left(\mathrm{O}^{+2}, \mathrm{Ne}^{+2}, \mathrm{He}^{+}, \mathrm{He}^{+2}\right)$, the $T_{e}\left(S^{++}\right)$temperature for the intermediate-ionization zone $\left(S^{+2}, A r^{+2}\right)$, and the $T_{e}\left(O^{+}\right)$temperature for the lowionization zone $\left(\mathrm{O}^{+}, \mathrm{N}^{+}, \mathrm{S}^{+}, \mathrm{Fe}^{+2}\right)$. For sake of homogeneity, and because of the aforementioned potential problems affecting $T_{e}\left(O^{+}\right)$determinations, we computed the $S^{++}$and $\mathrm{O}^{+}$temperatures from the theoretical relations of Garnett (1992):

$T_{e}[S I I I]=0.83 \times T_{e}[O I I I]+1700 K$,

$T_{e}[O I I]=0.70 \times T_{e}[O I I I]+3000 K$.

To determine the abundances of the various ions, we used the extinction-corrected fluxes (listed in Table 2) of the following lines: He I $\lambda 4471$, He I $\lambda$ 5876, and He I $\lambda 6678$ for $\mathrm{He}^{+}$, He II $\lambda 4686$ (when available) for $\mathrm{He}^{+2}$, [N II] $\lambda 6584$ for $N^{+}$, [O III] $\lambda \lambda 4959,5007$ for $O^{+2}$, [O II] $\lambda \lambda 3726,29$ for $O^{+}$, [Ne III] $\lambda 3869$ for $N e^{+2}$, [S II] $\lambda \lambda 6716,31$ for $S^{+}$, [S III] $\lambda 9069$ for $S^{+2}$, and [Ar III] $\lambda 7136$ for $\mathrm{Ar}^{+2}$. The PyNeb code adopts the He I emissivities of Porter et al. (2012, 2013) including collisional excitation, so no correction to the emission line fluxes for this effect (Clegg 1987) needs to be applied. The He I $\lambda 7065$ line, which has a strong contribution from collisional excitation, was not included in the computation of the $\mathrm{He}^{+}$; in fact, the uncertainties on the derived $n_{e}$ values translate into large errors on the $\mathrm{He}^{+}$abundance due to the strong dependence of the He I $\lambda 7065$ emissivity on density (see e.g. Fig. 4 of Porter et al. 2012). We did not use the faint HeI $\lambda 4922$ line or the HeI $\lambda 5015$ line, blended to the much stronger [O III] $\lambda 5007$ emission. The ion abundance uncertainties were derived running the getIonAbundance task for ranges of temperatures, densities, and flux ratios within the $\pm 1 \sigma$ levels, and conservatively adopting the maximum excursion around the nominal abundance value as our error. In the case of $\mathrm{He}^{+}$, the abundance was computed by averaging the individual abundances from the HeI $\lambda 4471$, HeI $\lambda 5876$, and HeI $\lambda 6678$ lines.

Total element abundances were derived from the abundances of ions seen in the optical spectra using ionization correction factors (ICFs). For Oxygen, the total abundance was computed as $O / H=\left(O^{+}+O^{+2}\right) / H^{+}$; the contribution of $\mathrm{O}^{+3}$ to the total oxygen abundance is in fact expected to be $<1 \%$, since $O^{+} /\left(O^{+}+O^{+2}\right)>0.1$ in our Hil regions (Izotov et al. 2006). The He abundance was computed from 
Table 2. Reddening-corrected Emission Fluxes for H II regions in DDO 68.

\begin{tabular}{|c|c|c|c|c|c|c|}
\hline Line & Reg-1 & Reg-3 & Reg-4 & Reg-7 & Reg-8 & Reg-9 \\
\hline [O II] $\lambda 3727$ & $27 \pm 1$ & $63 \pm 5$ & $84 \pm 2$ & $51 \pm 7$ & $290 \pm 20$ & $150 \pm 20$ \\
\hline H10 ג3978 & $4.5 \pm 0.2$ & $4.0 \pm 0.6$ & - & $5.9 \pm 0.5$ & - & - \\
\hline He I $\lambda 3820$ & - & $2.8 \pm 0.2$ & - & - & - & - \\
\hline H9+He II $\lambda 3835$ & $6.2 \pm 0.3$ & $5.8 \pm 0.6$ & - & $5 \pm 2$ & - & - \\
\hline [Ne III] $\lambda 3869$ & $15.6 \pm 0.5$ & $12.3 \pm 0.8$ & $10 \pm 1$ & $7.3 \pm 0.2$ & - & - \\
\hline H8+He I $\lambda 3889$ & $19 \pm 1$ & $17 \pm 2$ & $13.2 \pm 0.7$ & $18 \pm 2$ & $18 \pm 2$ & - \\
\hline $\mathrm{H} \epsilon+\mathrm{He} \mathrm{I}+[\mathrm{Ne} \mathrm{III}] \lambda 3970$ & $19.1 \pm 0.7$ & $18 \pm 1$ & $12 \pm 1$ & $16.6 \pm 0.8$ & $16 \pm 2$ & - \\
\hline Н $\delta \lambda 4101$ & $26.4 \pm 0.9$ & $27 \pm 2$ & $26.4 \pm 0.2$ & $26.3 \pm 0.4$ & $26 \pm 2$ & $32 \pm 3$ \\
\hline $\mathrm{H} \gamma \lambda 4340$ & $48 \pm 2$ & $48 \pm 4$ & $47.7 \pm 0.6$ & $47.6 \pm 0.9$ & $45 \pm 2$ & $50 \pm 8$ \\
\hline [O III] $\lambda 4363$ & $5.8 \pm 0.2$ & $3.5 \pm 0.6$ & - & $1.7 \pm 0.1$ & - & - \\
\hline He I $\lambda 4471$ & $3.7 \pm 0.3$ & - & $4.0 \pm 0.2$ & $4.6 \pm 0.1$ & - & - \\
\hline He II (WR) $\lambda 4686$ & - & $8 \pm 2$ & - & - & - & - \\
\hline He II $\lambda 4686$ & $2.30 \pm 0.07$ & - & - & - & - & - \\
\hline $\mathrm{H} \beta \lambda 4861$ & $100 \pm 4$ & $100 \pm 7$ & $100 \pm 1$ & $100 \pm 2$ & $100 \pm 5$ & $100 \pm 1$ \\
\hline He I $\lambda 4922$ & $1.03 \pm 0.07$ & - & - & - & - & - \\
\hline [O III] $\lambda 4959$ & $63 \pm 3$ & $43 \pm 3$ & $25.4 \pm 0.9$ & $23.5 \pm 0.4$ & $20 \pm 1$ & $13 \pm 1$ \\
\hline [O III] $\lambda 5007$ & $181 \pm 8$ & $125 \pm 9$ & $73 \pm 2$ & $66 \pm 1$ & $61 \pm 4$ & $50 \pm 6$ \\
\hline He I $\lambda 5015$ & $2.3 \pm 0.1$ & - & - & - & - & - \\
\hline He I $\lambda 5876$ & $9.3 \pm 0.3$ & $9.8 \pm 0.5$ & $7.8 \pm 0.2$ & $9.5 \pm 0.2$ & $9 \pm 2$ & - \\
\hline [OI] $\lambda 6302$ & $0.50 \pm 0.04$ & - & - & $1.4 \pm 0.4$ & $6.3 \pm 0.5$ & - \\
\hline [S III] $\lambda 6312$ & $0.67 \pm 0.05$ & - & - & - & - & - \\
\hline $\mathrm{H} \alpha \lambda 6563$ & $281 \pm 10$ & $290 \pm 20$ & $281 \pm 3$ & $280 \pm 4$ & $270 \pm 10$ & $300 \pm 30$ \\
\hline [N II] $\lambda 6584$ & $1.0 \pm 0.2$ & $2.3 \pm 0.1$ & $2.7 \pm 0.4$ & $1.9 \pm 0.1$ & $8.0 \pm 0.4$ & - \\
\hline He I $\lambda 6678$ & $2.7 \pm 0.2$ & $2.8 \pm 0.2$ & $2.8 \pm 0.1$ & $2.9 \pm 0.2$ & $2.5 \pm 0.3$ & - \\
\hline [S II] $\lambda 6716$ & $2.1 \pm 0.1$ & $4.8 \pm 0.6$ & $8 \pm 3$ & $4.9 \pm 0.1$ & $24 \pm 1$ & $14 \pm 2$ \\
\hline [S II] $\lambda 6731$ & $1.5 \pm 0.1$ & $3.6 \pm 0.2$ & $3.8 \pm 0.8$ & $3.7 \pm 0.4$ & $18 \pm 1$ & $6 \pm 1$ \\
\hline He I $\lambda 7065$ & $2.3 \pm 0.1$ & $2.5 \pm 0.2$ & $2.4 \pm 0.2$ & $1.8 \pm 0.1$ & - & - \\
\hline [Ar III] $\lambda 7136$ & $1.7 \pm 0.1$ & $1.9 \pm 0.1$ & $1.5 \pm 0.1$ & $1.4 \pm 0.4$ & - & - \\
\hline He I $\lambda 7281$ & $0.42 \pm 0.02$ & - & - & - & - & - \\
\hline [O II] $\lambda 7320$ & $0.67 \pm 0.08$ & - & - & - & $3.2 \pm 0.3$ & - \\
\hline [O II] $\lambda 7330$ & $0.5 \pm 0.1$ & - & - & - & $3.9 \pm 0.6$ & - \\
\hline P10 $\lambda 9017$ & $21.3 \pm 0.7$ & - & - & - & - & - \\
\hline [S III] $\lambda 9069$ & $3.5 \pm 0.2$ & $3.2 \pm 0.3$ & $3 \pm 1$ & $2.4 \pm 0.7$ & $3.5 \pm 0.2$ & - \\
\hline P9 $\lambda 9229$ & $2.25 \pm 0.07$ & $2.4 \pm 0.4$ & - & - & - & - \\
\hline [S III] $\lambda 9532$ & $7.8 \pm 0.4$ & $8 \pm 1$ & - & - & - & - \\
\hline P8 $\lambda 9547$ & $3.2 \pm 0.2$ & - & - & - & - & - \\
\hline $\mathrm{E}(\mathrm{B}-\mathrm{V})$ & $0.08 \pm 0.01$ & $0.12 \pm 0.01$ & $0.07 \pm 0.01$ & $0.08 \pm 0.01$ & $0.24 \pm 0.02$ & $0.1 \pm 0.1$ \\
\hline $\mathrm{F}(\mathrm{H} \beta)\left[10^{-15} \mathrm{erg} / \mathrm{s} / \mathrm{cm}^{2}\right]$ & $3.3 \pm 0.1$ & $1.6 \pm 0.1$ & $0.78 \pm 0.01$ & $0.74 \pm 0.01$ & $1.11 \pm 0.07$ & $0.14 \pm 0.05$ \\
\hline v helio $[\mathrm{km} / \mathrm{s}]$ & $513 \pm 3$ & $522 \pm 1$ & $508 \pm 4$ & $479 \pm 1$ & $487 \pm 6$ & $519 \pm 7$ \\
\hline
\end{tabular}

Notes: Fluxes are given on a scale where $F(H \beta)=100$.

$\mathrm{He}^{+}$, with the exception of Reg. 1 where we added the contribution from $\mathrm{He}^{+2}$. On the other hand, the contribution from the broad He II $\lambda 4686$ emission detected in Reg. 3 was not included, since likely of stellar origin. For all the elements other than $\mathrm{O}$ and $\mathrm{He}$, we adopted the ICFs from Izotov et al. (2006) for the low Z regime (Eq. (18) through (22) in that paper) to obtain total abundances.

As mentioned in Section 2, the abundances of Reg. 1 and Reg. 3 (as well as those of Reg. 2 and Reg. 6, not present in our sample) were inferred with the direct $T_{e}$ method by other authors (Pustilnik et al. 2005, 2008; Izotov \& Thuan 2007, 2009). For these two regions, our abundances are consistent with theirs: more specifically, we obtain for Reg. 1 an abundance of $12+\log (\mathrm{O} / \mathrm{H})=7.10 \pm 0.02$, within $1 \sigma$ of the value of $7.14 \pm 0.03$ from Izotov \& Thuan (2007) and within 2 $\sigma$ of the value of $7.19 \pm 0.05$ from Pustilnik et al. (2008); for Reg. 3 we derive $12+\log (\mathrm{O} / \mathrm{H})=7.14 \pm 0.07$, within $1 \sigma$ of the values of $7.15 \pm 0.04$ and $7.10 \pm 0.06$ from Izotov \& Thuan (2009) and Pustilnik et al. (2008), respectively. We therefore confirm an extremely low oxygen abundance in DDO 68' s H II regions. Furthermore, we report for the first time a 3- $\sigma$ direct method abundance measurement in a region in the "tail" of DDO 68, namely Reg. 7 (since Pustilnik et al. (2005) reported there a $1-\sigma$ [O III $] \lambda 4363$ detection), finding an abundance of $12+\log (\mathrm{O} / \mathrm{H})=6.96 \pm 0.09$, even lower than in the "head" of the comet. Our results are summarized in Table 3.

\subsection{Indirect method}

Since [O III] $\lambda 4363$ was measured only in half of our targets, for all the $\mathrm{H}$ II regions in our sample we also derived oxygen abundances using the indirect method. As widely discussed in the literature, this method is affected by much larger uncertainties than the direct one, and furthermore different calibrations can disagree by as much as $\sim 0.5$ dex (Kewley \& Ellison 2008). For our study, we considered the two different calibrations proposed by Yin et al. (2007) and 
Table 3. Derived Properties for H II regions in DDO 68.

\begin{tabular}{lcccc}
\hline Line & Reg-1 & Reg-3 & Reg-7 & Reg-8 \\
\hline$n_{e}\left[\mathrm{~cm}^{-3}\right]$ & $\leq 26$ & $76_{-76}^{+169}$ & $116_{-116}^{+148}$ & $40_{-2}^{+2}$ \\
$T_{e}\left(\mathrm{O}^{+}\right)[\mathrm{K}]$ & $23000 \pm 3000$ & - & - & $13000 \pm 1000$ \\
$T_{e}\left(\mathrm{O}^{++}\right)[\mathrm{K}]$ & $19400 \pm 400$ & $18000 \pm 2000$ & $17000 \pm 2000$ & - \\
$T_{e}\left(\mathrm{~S}^{++}\right)[\mathrm{K}]$ & $19000 \pm 1000$ & - & - & - \\
\hline$\left(\mathrm{O}^{+} / \mathrm{H}^{+}\right) \times 10^{6}$ & $1.66 \pm 0.08$ & $5 \pm 1$ & $4 \pm 1$ & - \\
$\left(\mathrm{O}^{++} / \mathrm{H}^{+}\right) \times 10^{6}$ & $10.9 \pm 0.5$ & $9 \pm 2$ & $5 \pm 1$ & - \\
$12+\log (\mathrm{O} / \mathrm{H})$ & $7.10 \pm 0.02$ & $7.14 \pm 0.07$ & $6.96 \pm 0.09$ & - \\
\hline$H e / H$ & $0.082 \pm 0.003$ & $0.079 \pm 0.002$ & $0.085 \pm 0.010$ & - \\
$Y$ & $0.246 \pm 0.007$ & $0.240 \pm 0.005$ & $0.25 \pm 0.02$ & - \\
\hline$\left(N^{+} / H^{+}\right) \times 10^{7}$ & $0.7 \pm 0.1$ & $1.8 \pm 0.3$ & $1.5 \pm 0.1$ & - \\
$I C F\left(N^{+}\right)$ & 7.06 & 2.92 & 2.27 & - \\
$12+\log (\mathrm{N} / H)$ & $5.70 \pm 0.06$ & $5.72 \pm 0.06$ & $5.54 \pm 0.04$ & - \\
$\log (\mathrm{N} / \mathrm{O})$ & $-1.39 \pm 0.07$ & $-1.4 \pm 0.1$ & $-1.4 \pm 0.1$ & - \\
\hline$\left(\mathrm{Ne} e^{++} / H^{+}\right) \times 10^{6}$ & $2.1 \pm 0.1$ & $2.1 \pm 0.4$ & $1.3 \pm 0.4$ & - \\
$I C F\left(\mathrm{Ne} e^{++}\right)$ & 1.06 & 1.15 & 1.19 & - \\
$12+\log (\mathrm{Ne} / H)$ & $6.35 \pm 0.02$ & $6.37 \pm 0.09$ & $6.2 \pm 0.1$ & - \\
$\log (\mathrm{Ne} / \mathrm{O})$ & $-0.75 \pm 0.03$ & $-0.8 \pm 0.1$ & $-0.8 \pm 0.2$ & - \\
\hline$\left(S^{+} / H^{+}\right) \times 10^{7}$ & $0.31 \pm 0.02$ & $0.8 \pm 0.1$ & $0.9 \pm 0.1$ & - \\
$\left(S^{++} / H^{+}\right) \times 10^{7}$ & $2.0 \pm 0.1$ & $2.1 \pm 0.2$ & $1.6 \pm 0.5$ & - \\
$I C F\left(S^{+}+S^{+}++\right)$ & 1.74 & 1.02 & 0.93 & - \\
$12+\log (\mathrm{S} / H)$ & $5.61 \pm 0.02$ & $5.47 \pm 0.04$ & $5.36 \pm 0.09$ & - \\
$\log (\mathrm{S} / \mathrm{O})$ & $-1.49 \pm 0.03$ & $-1.67 \pm 0.08$ & $-1.6 \pm 0.1$ & - \\
\hline$\left(\mathrm{Ar}++/ H^{+}\right) \times 10^{7}$ & $0.47 \pm 0.03$ & $0.59 \pm 0.08$ & $0.4 \pm 0.1$ & - \\
$I C F(\mathrm{Ar})$ & 1.26 & 1.08 & 1.07 & - \\
$12+\log (\mathrm{Ar} / H)$ & $4.77 \pm 0.03$ & $4.80 \pm 0.06$ & $4.7 \pm 0.1$ & - \\
$\log (\mathrm{Ar} / \mathrm{O})$ & $-2.33 \pm 0.03$ & $-2.34 \pm 0.09$ & $-2.3 \pm 0.1$ & - \\
\hline \hline
\end{tabular}

Table 4. Oxygen abundances for $\mathrm{H}$ II regions in DDO 68 from direct and indirect methods.

\begin{tabular}{lccc}
\hline \hline Region & \multicolumn{3}{c}{$12+\log (\mathrm{O} / \mathrm{H})$} \\
\hline & $T_{e}$-Direct & Yin et al. 07 & Pilyugin \& Grebel 2016 \\
\hline 1 & $7.10 \pm 0.02$ & $7.09 \pm 0.02$ & $7.10 \pm 0.06$ \\
3 & $7.14 \pm 0.07$ & $7.00 \pm 0.05$ & $7.09 \pm 0.04$ \\
7 & $6.96 \pm 0.09$ & $6.69 \pm 0.03$ & $6.69 \pm 0.04$ \\
8 & - & $7.28 \pm 0.06$ & $7.18 \pm 0.04$ \\
9 & - & $7.0 \pm 0.3$ & - \\
4 & - & $6.85 \pm 0.01$ & $6.83 \pm 0.04$ \\
\hline \hline
\end{tabular}

Pilyugin \& Grebel (2016); we caution that these relations have been calibrated on direct-method abundances of $12+$ $\log (\mathrm{O} / \mathrm{H}) \gtrsim 7.10$, whereas we are applying them to a lower metallicity regime for our study of DDO 68. Figure 3 shows, for Reg. 1, Reg. 3, and Reg. 7 , the comparison between the abundances derived with the direct method and those obtained with the two different indirect-method calibrations. We notice that for Reg. 1 and Reg. 3 the Pilyugin \& Grebel calibration provides the best agreement with the direct- $\mathrm{T}_{\boldsymbol{e}}$ method. On the other hand, both calibrations underestimate by more than 0.2 dex the abundance derived for Reg. 7 , the most metal-poor. The results from the two calibrations for our six H II regions are summarized in Table 4.

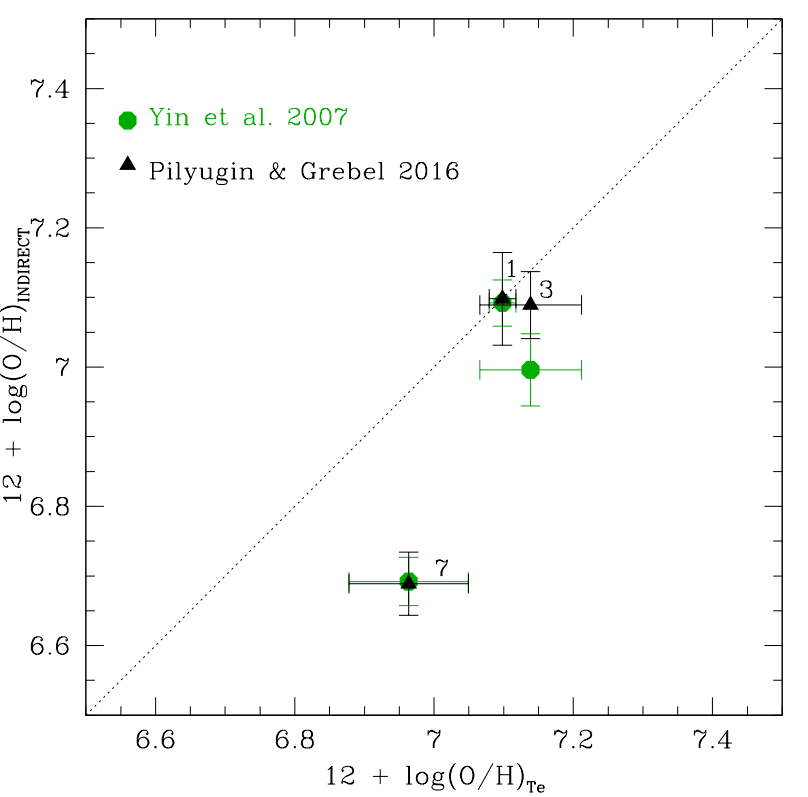

Figure 3. Oxygen abundances derived with the direct $T_{e}$ method and with different indirect-method calibrations (Yin et al. 2007; Pilyugin \& Grebel 2016) for Reg. 1, Reg. 3, and Reg. 7. The dotted line is the one-to-one relation. 


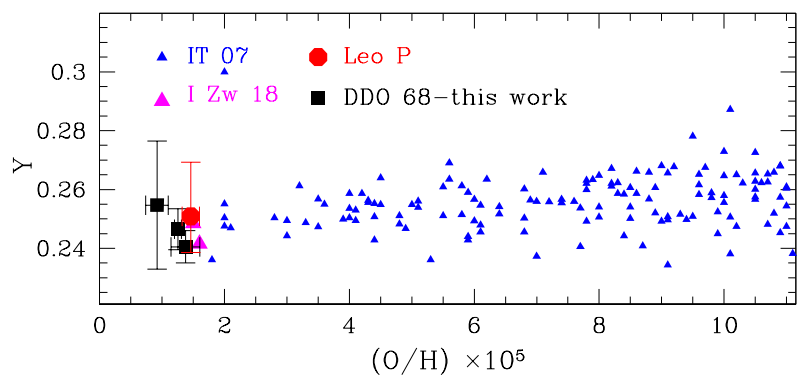

Figure 4. Helium mass fraction as a function of the oxygen abundance by number in DDO 68's H II regions (black squares) compared with literature values for other metal-poor star-forming galaxies. The blue triangles are the low-metallicity $\mathrm{H}$ II regions galaxies from Izotov et al. (2007), where we have highlighted the value inferred for IZw18 with the large magenta triangle; the large red circle corresponds to the helium mass fraction inferred by Skillman et al. (2013) for Leo P.

\section{ABUNDANCES AND ABUNDANCE RATIOS OF H II REGIONS}

As described in Section 2 and shown in Figure 1, Reg. 8 is close to the galaxy centre, Reg. 7 and Reg. 9 lie on the cometary tail that is often considered the accreted satellite of DDO 68, while Reg. 1, Reg. 3 and Reg. 4 design the ring-like structure which might be either the head of the comet/satellite or the periphery of the main body. We can thus try to use the abundances listed in Table 3 to get information on the evolutionary status of DDO 68, in terms either of a single galaxy or of two merged bodies.

\subsection{Helium}

Since the pioneering work by Peimbert \& Torres-Peimbert (1974), the helium abundance derived from spectroscopy of the $\mathrm{H}$ II regions in low-metallicity star-forming dwarfs has been considered as the best tool in the quest of the primordial helium abundance, and therefore an important test of the predictions of standard big bang nucleosynthesis (SBBN, e.g., Yang et al. 1984). However, to make inferences on the SBBN, the primordial helium abundance must be inferred with an accuracy better than one percent, which is highly challenging, as widely discussed in the literature (e.g. Olive \& Skillman 2004; Izotov et al. 2007; Aver et al. 2011). XMPs are extremely valuable for the determination of the primordial helium abundance, since the extrapolation of the $\mathrm{He} / \mathrm{H}$ vs $\mathrm{O} / \mathrm{H}$ distribution, or its mass fraction version $\Delta \mathrm{Y} / \Delta \mathrm{Z}$, to zero metallicity is minimal. For instance, from their samples of $\mathrm{H}$ II regions in low-metallicity galaxies, Izotov et al. (2014) find $\mathrm{Y}_{P}=0.255 \pm 0.002$ (statistical + systematic error), Aver et al. (2013) infer $\mathrm{Y}_{P}=0.2465 \pm$ 0.0097, and Fernández et al. (2018) find $\mathrm{Y}_{P}=0.245 \pm 0.007$, marginally consistent with each other within the errors.

The extremely low oxygen abundance of DDO 68 makes it a key object in the definition of the actual slope of $\mathrm{He} / \mathrm{H}$ with $\mathrm{O} / \mathrm{H}$, that has been the subject of hot debates over decades (see, e.g., Izotov et al. 2007; Peimbert et al. 2007, and references therein). What is measured from the $\mathrm{H}$ II region spectra is the abundance by number of helium relative to hydrogen, whereas the primordial helium abundance is usually estimated in mass fraction $\mathrm{Y}_{P}$. We then convert our measured $\mathrm{He} / \mathrm{H}$ values to the corresponding mass fraction Y via Pagel et al. (1992)'s simple relation:

$Y=\{4 \mathrm{He} / \mathrm{H}[1-20(\mathrm{O} / \mathrm{H})]\} /(1+4 \mathrm{He} / \mathrm{H})$.

We infer helium mass fractions of $\mathrm{Y}=0.246 \pm 0.007$, $0.240 \pm 0.005$, and $0.25 \pm 0.02$ for Regions 1,3 and 7, respectively. Notice that the quoted errors do not include systematic uncertainties due to the underlying theoretical assumptions and adopted physical parameters in the abundance computation. Peimbert et al. (2007), and references therein, evaluated the consequences on the derived primordial helium of several effects, including new atomic data. Izotov et al. (2007) extensively discussed all possible systematic effects that challenge high-accuracy determinations of helium abundances in H II regions: He emissivities, reddening, temperature and ionization structure of the H II region, underlying stellar He absorption, collisional excitation of hydrogen lines, deviation from case B recombination. Using a Markov Chain Monte Carlo analysis, Aver et al. (2011) demonstrated that, even in presence of high-quality spectra of H II regions, statistical and systematical uncertainties in the derived helium abundances can be as high as several percents.

Figure 4 shows how DDO 68's helium abundances derived here compare with the distribution of $\mathrm{Y}$ vs $\mathrm{O} / \mathrm{H}$ in metal-poor star-forming galaxies. Our helium mass fractions lie at the low-oxygen end of the plot, and cover a range of values from slightly lower than in IZw18 (Izotov et al. 2007), to slightly higher than in Leo P (Skillman et al. 2013), with the warning that the highest value shows a significantly large error. DDO 68's helium abundances are consistent with Izotov et al. (2014)'s, Aver et al. (2011)'s and Fernández et al. (2018)'s primordial values within $3 \sigma$ uncertainties. We notice that Fernández et al. (2018) excluded from their linear regression the regions with higher $\mathrm{He} / \mathrm{H}$ and $\mathrm{N} / \mathrm{O}$, arguing that they could be contaminated by nearby Wolf-Rayet stars. For this reason we do not attempt to derive a new regression line including also our data points, but simply show them in the plot.

Only in the last ten years or so, have people been able to compare the predictions of SBBN with other stringent constraints, resulting from the estimates of the baryon density from the satellites devoted to measuring the cosmic background radiation, WMAP (Wilkinson Microwave Anisotropy Probe, Spergel et al. 2003) and the more recent Planck mission (Planck Collaboration et al. 2011). Under the assumption of SBBN and of a neutron lifetime of $\sim 880 \mathrm{~s}$ (Beringer et al. 2012), the baryon densities provided by the WMAP or the Planck experiments imply a primordial helium abundance of $\mathrm{Yp}=0.2467 \pm 0.0006$ (Spergel et al. 2007; Planck collaboration XIII 2016). The He abundances determined by us for DDO 68 are therefore compatible with the primordial value from WMAP or Planck within the quoted uncertainties.

\subsection{Spatial distribution of oxygen abundance}

We have analysed the spatial distribution of the oxygen abundances inferred for our H II regions looking for signatures (or lack thereof) of the interactions with the galaxy's satellites. Table 4 and Figure 5 show that there is no clear 


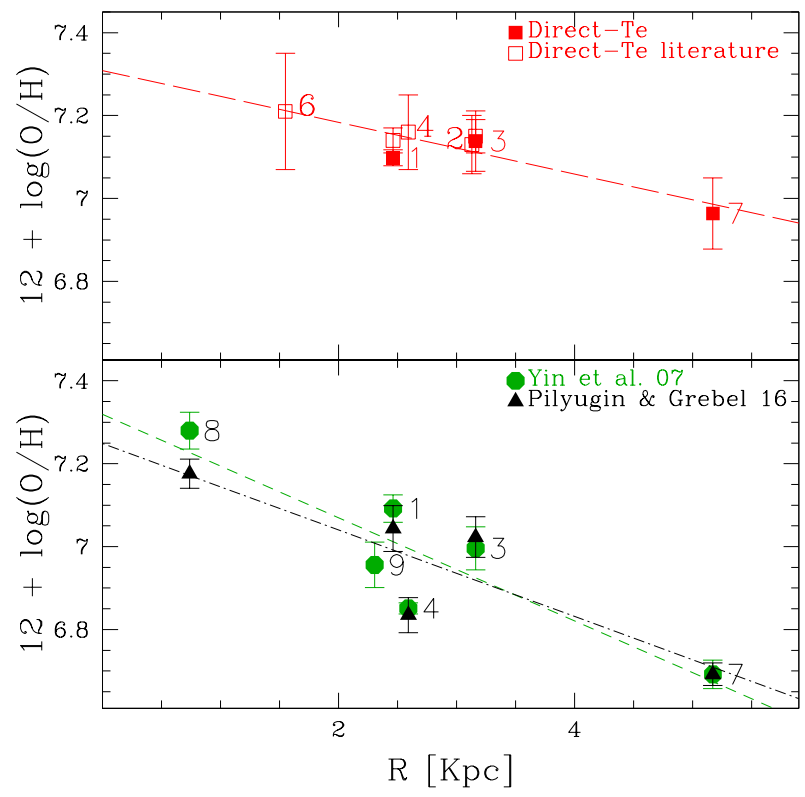

Figure 5. Top panel: oxygen abundance as a function of the distance from the centre of DDO 68 derived with the direct $\mathrm{T}_{e}$ method; full squares indicate our results, while open squares are the abundance values from Izotov \& Thuan (2007) and Izotov \& Thuan (2009). The dashed line is the linear fit to all values. Bottom panel: oxygen abundance as a function of the distance derived in this work with the Yin et al. (2007) and Pilyugin \& Grebel (2016)'s indirect-method calibrations. The dashed and dot-dashed lines are the separate fits to the values obtained with the Yin et al. and Pilyugin \& Grebel calibrations, respectively.

evidence for the presence of a metallicity dichotomy in DDO 68, which could indicate a recent merging between two systems with very different initial metallicities. In particular, from an analysis of the color-magnitude diagram of the resolved stars, Tikhonov, Galazutdinova, \& Lebedev (2014) claimed the presence of two different metallicity components of $\sim 1 / 4$ and $\sim 1 / 16$ solar, belonging to the main system and to the accreted companion, respectively. However, from our $\mathrm{H}$ II region analysis we do not find compelling evidence for two distinct metallicity populations in DDO 68, and in particular we do not detect a component as high in metallicity as $\sim 1 / 4$ solar (although there is no direct $\mathrm{O}$ abundance measurement in the most internal Reg. 8).

Rather than two separate metallicity populations, our analysis suggests the presence of a "smooth" trend of decreasing metallicity from the galaxy center outwards. This is observed both for the direct-method abundance sample and for the indirect-method one. In Figure 5 the oxygen abundance has been plotted as a function of the distance from the galaxy centre, defined as the point of maximum light density (inferred from our HST and LBT imaging) and indicated by a cross in Figure 1. The top panel of Fig. 5 shows the abundance results obtained in this work with the direct$\mathrm{T}_{e}$ method, complemented by direct-method abundances of DDO 68 from the literature (Izotov \& Thuan 2007, 2009). The linear fit to the combined sample provides a slope of $-0.06 \pm 0.03 \mathrm{dex} / \mathrm{kpc}$. In the bottom panel of Fig. 5 we show instead the results obtained in this work with the Yin et al.
(2007) and Pilyugin \& Grebel (2016)'s indirect-method calibrations (see Section 4.1). The separate fits to the Yin et al. and Pilyugin \& Grebel abundance sets provides slopes of $-0.12 \pm 0.03 \mathrm{dex} / \mathrm{kpc}$ and $-0.10 \pm 0.04 \mathrm{dex} / \mathrm{kpc}$, steeper than the direct-method. Notice that the steeper gradients obtained with the indirect methods may be due to a poor calibration of the empirical relations at $12+\log (\mathrm{O} / \mathrm{H})<7.1$, as already discussed in Section 4.1. In conclusion, we get evidence for a negative metallicity gradient in DDO 68, with $\Delta \log (\mathrm{O} / \mathrm{H}) / \Delta \mathrm{R}$ in the range -0.06 to $-0.12 \mathrm{dex} \mathrm{kpc}^{-1}$, and no obvious chemical signature of a merging in our data.

In the past, metallicity gradients were considered quite small, or even absent, in dwarf galaxies (see, e.g., Skillman et al. 2013, and references therein). However, until recently, very few, sometimes only one, H II regions were usually observed in galaxies outside the Local Group. Furthermore, uncertainties on the derived abundances could be quite large in absence of high signal-to-noise spectra. Gradients have been found in star-forming dwarfs when modern multi-object spectrographs have allowed people to measure with significantly improved accuracy several H II regions per galaxy (Annibali et al. 2015; Pilyugin et al. 2015; Annibali et al. 2017), which suggests that others may have gone undetected for lack of appropriate data rather than for actual absence.

For comparison with other literature studies it is useful to express the metallicity gradient in DDO 68 as a function of the isophotal radius $R_{25}$. Adopting $R_{25}=1.3^{\prime}$ from de Vaucouleurs et al. (1991) and $\mathrm{D}=12.7 \mathrm{Mpc}$ from Sacchi et al. (2016), we obtain $R_{25}=4.95 \mathrm{kpc}$; the metallicity gradients are therefore -0.30 dex $R_{25}^{-1}$ (direct method) and $-0.59 \operatorname{dex} R_{25}^{-1}$ (indirect method). For comparison, the metallicity gradients obtained with the direct method in the dwarfs NGC 4449 and NGC 1705 are $-0.29 \pm 0.08$ $\operatorname{dex} R_{25}^{-1}$ (Annibali et al. 2017) and $-0.33 \pm 0.08 \operatorname{dex} R_{25}^{-1}$ (Annibali et al. 2015), similar to the value obtained for DDO 68. Using a combination of direct and indirect-method abundances, Pilyugin et al. (2015) found gradients in the range $-0.04 \pm 0.04$ to $-0.36 \pm 0.11$ dex $R_{25}^{-1}$ for a sample of 14 irregular galaxies, compatible with our directmethod gradient of DDO 68, but significantly flatter than the value obtained with the indirect method. Spiral galaxies display gradients too; using the indirect method calibration of Pilyugin et al. (2012), Zinchenko et al. (2016) found, for a sample of 88 disc galaxies in the CALIFA survey, gradients in the range between -0.07 and $-0.30 \operatorname{dex} R_{25}^{-1}$, with a few exceptions with gradient $-0.64 \operatorname{dex} R_{25}^{-1}$. Thus, the metallicity gradient in DDO 68 is consistent with the steepest values derived in spiral galaxies.

\subsection{Abundance Ratios}

Figure 6 shows the distribution of the abundance ratios $\mathrm{Ne} / \mathrm{O}, \mathrm{S} / \mathrm{O}$ and $\mathrm{Ar} / \mathrm{O}$ as a function of the oxygen abundance (direct method). The black squares correspond to the values we have derived for DDO 68's H in regions, while the other symbols show the corresponding literature values for other metal-poor dwarfs. Neon, sulfur, and argon are all produced by the same massive stars that produce oxygen; hence, their abundance ratios to oxygen are expected to be independent of the oxygen abundance. This is indeed the case 
also in DDO 68, whose ratios lie all on the lower oxygen tail of the flat distribution inferred for the other dwarfs (see references in the caption to Fig. 6). In this respect, although extremely oxygen-poor, DDO 68 behaves well, following the same average trend as the other galaxies.

Vice versa, DDO 68 does not appear to follow the general trend for what concerns nitrogen. Figure 7 shows the $\mathrm{N} / \mathrm{O}$ ratio vs $\mathrm{O} / \mathrm{H}$ in our $\mathrm{H}$ II regions (black filled squares) and in those of the same large sample of star-forming dwarf galaxies plotted in Fig. 6 . While the very metal poor galaxy IZw 18 , with its $\log (\mathrm{N} / \mathrm{O}) \sim-1.6$, lies exactly on the tight plateau identified by Izotov \& Thuan (1999) for XMPs (i.e., galaxies with $12+\log (\mathrm{O} / \mathrm{H}) \leq 7.2)$, all the DDO 68' $\mathrm{s}$ $\mathrm{H}$ il regions have even lower oxygen but significantly higher $\mathrm{N} / \mathrm{O}$, thus suggesting a hint of reverse trend with respect to that described by less metal-poor galaxies.

The $\mathrm{N} / \mathrm{O}$ vs $\mathrm{O} / \mathrm{H}$ diagram has been used to discuss the primary vs secondary nature of nitrogen for decades. A primary element is one (like oxygen) that can be synthesised in stars directly from primordial compositions, whereas a secondary element can be produced only in stars that at birth already contain seed/catalyst elements heavier than helium. As described by, e.g., Tinsley (1980, and references therein), the canonical prediction for the abundances of secondary elements is that they increase as the square of primary abundances; hence, a secondary/primary abundance ratio is expected to scale linearly with the primary element abundance. The circumstance that $\mathrm{N} / \mathrm{O}$ seems proportional to $\mathrm{O} / \mathrm{H}$ in all galaxies with $12+\log (\mathrm{O} / \mathrm{H}) \geq 7.5-8.0$ (e.g., Pagel 1985; van Zee et al. 1998), led people to conclude that nitrogen is a secondary element, a conclusion in agreement with standard stellar nucleosynthesis prescriptions that require catalyst carbon to produce N. However, already forty years ago, Lequeux et al. (1979) argued that the behaviour of the N/O ratios observed in a few irregular and blue compact galaxies should be ascribed to a non negligible fraction of primary nitrogen. Indeed, in 1981, Renzini \& Voli (1981) showed that nitrogen can be synthesised as a primary element in favourable conditions, namely, when deep convection brings the seeds necessary for the nitrogen production to the appropriate stellar layer, even if they were not present in the original composition but were synthesised in the stellar core during earlier evolutionary phases. In practice, they demonstrated that, in the Hot Bottom Burning (HBB) evolutionary phase of intermediate mass stars, nitrogen is synthesised during their hydrogen-envelope burning, using as catalyst the carbon previously produced in the core of the same star. The existence of a primary (minority) fraction of $\mathrm{N}$ was confirmed by the combination of chemical evolution models with $\mathrm{H}$ II region observations in the Milky Way and other spiral and late-type galaxies (e.g., Matteucci \& Tosi 1985; Diaz \& Tosi 1986; Vila-Costas \& Edmunds 1993; Marconi et al. 1994). In particular, it was shown that N/O is both observed and predicted to be rather flat across individual galaxies even in the oxygen-rich regime, although it increases with $\mathrm{O} / \mathrm{H}$ from one galaxy to the other.

A further challenge to the interpretation of nitrogen's nature came from the $\mathrm{N} / \mathrm{O}$ vs $\mathrm{O} / \mathrm{H}$ diagram when people (see, e.g., Garnett 1990; Kobulnicky \& Skillman 1996; Izotov \& Thuan 1999) suggested that in the lower oxygen regime $\log (\mathrm{N} / \mathrm{O})$ shows a plateau. In particular,
Izotov \& Thuan (1999) proposed that XMPs have a plateau at $\log (\mathrm{N} / \mathrm{O}) \simeq-1.6$. Even invoking a significant fraction of primary nitrogen from $\mathrm{HBB}$, this evidence was difficult to reconcile with standard nucleosynthesis predictions where oxygen is enriched much earlier than nitrogen because it is produced by massive, short-lived stars, while $\mathrm{N}$ should come from intermediate-mass, longer-lived ones. As a way out of this issue, Izotov \& Thuan (1999) suggested that nitrogen could not only be primary but also be produced by low-metallicity massive stars, as partly envisaged by Timmes, Woosley, \& Weaver (1995). Indeed, Maeder \& Meynet (2001) new stellar evolution models with rotation provided a theoretical support to this suggestion, since they predicted significant nitrogen enhancements in massive stars, with the $\mathrm{N}$ excesses being larger at larger stellar luminosities and at lower metallicity.

Now, we might be facing a new challenge, since the $\log (\mathrm{N} / \mathrm{O})$ we derived in all DDO 68's H II regions is definitely higher than -1.6. The interesting point is that DDO 68 is not a lonely outlier, but shows almost exactly the same high ratio (red circle in Fig. 7) inferred for Leo P (Skillman et al. 2013), another XMP, and for few other low-metallicity systems (see e.g., Nava et al. 2006; van Zee \& Haynes 2006; Izotov et al. 2009). Skillman et al. (2013) discussed at length the apparent inconsistency of Leo P's N/O with Izotov \& Thuan's plateau, posing key questions, but without reaching firm conclusions: is there actually a plateau or is it there a significant scatter (whether intrinsic or observational) as suggested by Garnett (1990)? Are there systematic differences between emission line galaxies and relatively quiescent dwarfs? Does Leo $\mathrm{P}$ show high $\mathrm{N} / \mathrm{O}$ because it is observed during a long period of relative quiescence in which $\mathrm{N}$ enriches the medium but $\mathrm{O}$ does not, as in the time delay scenario proposed by Garnett (1990)?

We do not have conclusions either, but we agree with them that in DDO 68, as in Leo P, the current SF activity, although present, is much lower than in the XMPs that defined Izotov \& Thuan (1999) plateau (IZw18, SBS1415+437, SBS0335-052). Its has been suggested (e.g., Pilyugin 1992; Henry et al. 2000) that the observed N/O ratio can be used as a "clock" to indicate the time since the most recent burst of star formation, due to the delayed release of $\mathrm{N}$ into the ISM compared to O. Since the highest activity of SF in DDO 68 has occurred between $\sim 10$ and $\sim 300$ Myr ago (Sacchi et al. 2016), is it possible that the high N/O ratio measured there is due to a substantial nitrogen enrichment from the population of intermediate-mass stars, compared to a relatively lower oxygen enrichment from the less active recent SF?

Or, given the strong dependence on rotational velocity and metallicity of the nitrogen primary production in massive stars (Maeder \& Meynet 2001), could it be that in these XMPs $\mathrm{N}$ is actually produced in stars more massive than the O-producers, and is thus released to the surrounding medium earlier than $\mathrm{O}$ ? In other words, could we invoke a reversed time-delay model in XMPs, with N/O decreasing for increasing $\mathrm{O} / \mathrm{H}$ ? The most conservative option would be to attribute the high $\mathrm{N} / \mathrm{O}$ (and the high $\mathrm{He} / \mathrm{H}$ ) to the presence of Wolf-Rayet stars close to our H II regions, following the argument by Fernández et al. (2018). Indeed, a broad He II $\lambda 4686$ feature, possibly due to the presence of WR stars, is detected in Reg.3. 


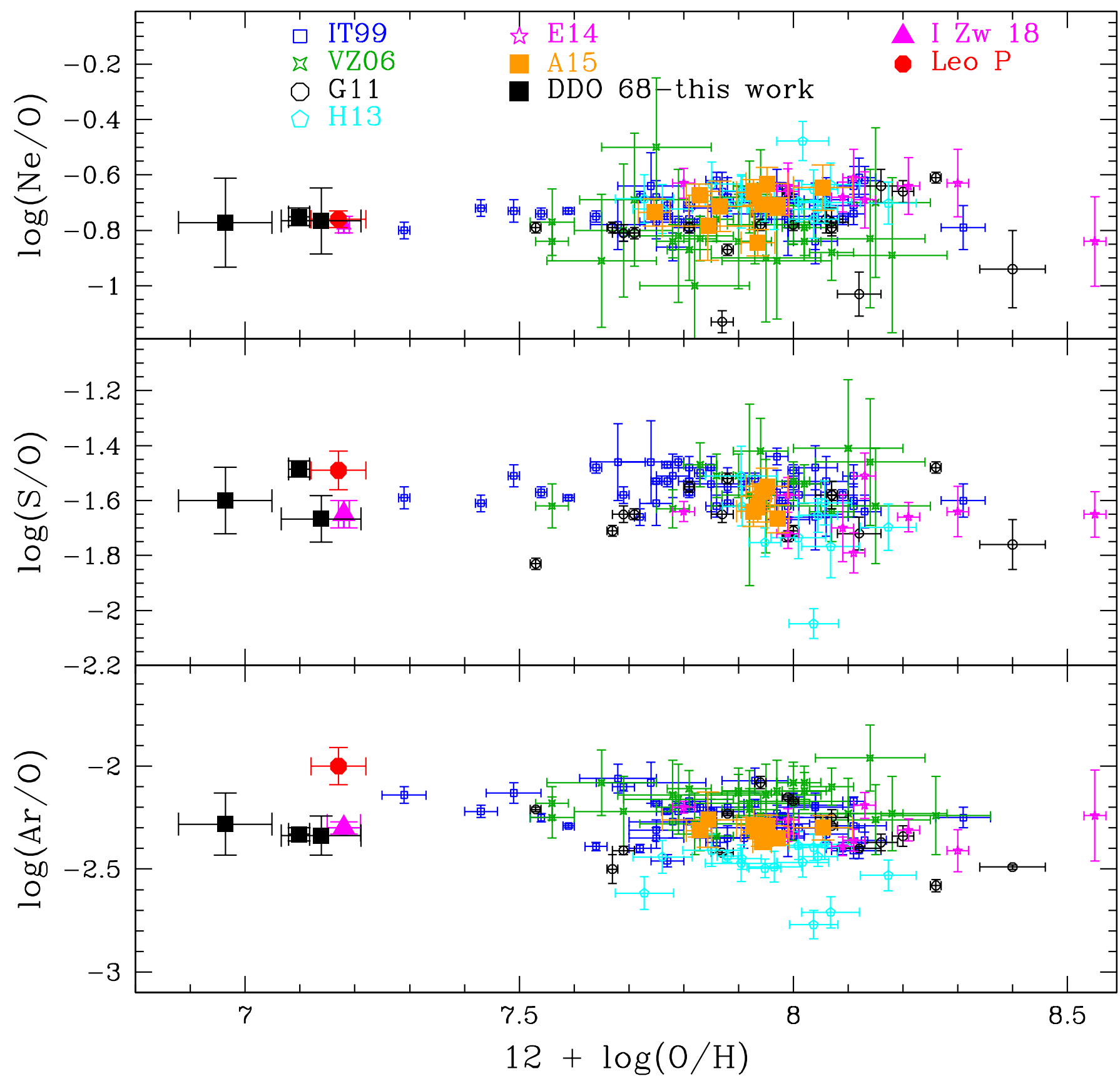

Figure 6. Chemical abundance ratio over oxygen of neon (top panel), sulfur (centre), and argon (bottom). The black filled squares show the ratios inferred for DDO 68 in this paper, while the other symbols show the literature values for other star forming dwarfs: IT99 (Izotov \& Thuan 1999); VZ06 (van Zee \& Haynes 2006); G11 (Guseva et al. 2011); H13 (Haurberg et al. 2013 ); E14 (Esteban et al. 2014); A15 (Annibali et al. 2015). We have emphasized with larger symbols the ratios in IZw18 (magenta triangle; Izotov \& Thuan 1999) and in LeoP (red circle; Skillman et al. 2013). 


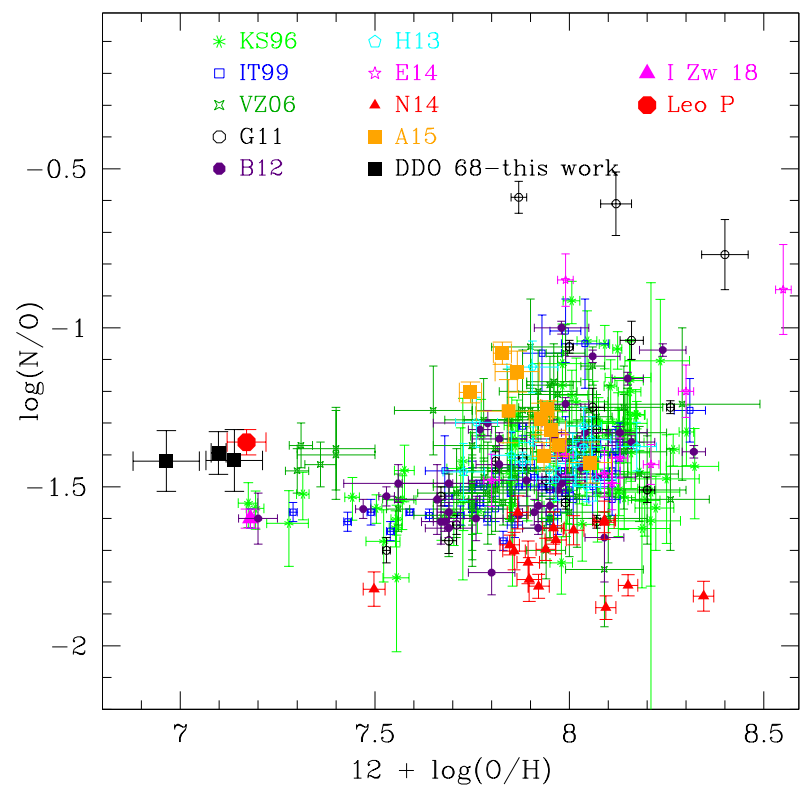

Figure 7. Nitrogen over oxygen abundance ratio as a function of the oxygen abundance. Symbols are as in Figure 6.

\section{RADIAL VELOCITIES}

Radial velocities were derived from the most prominent emission lines in the spectra. We derived mean velocities averaging the $\mathrm{H} \delta, \mathrm{H} \gamma, \mathrm{H} \beta$ and [O III] $\lambda \lambda 44959,5007$ lines in the blue MODS channel, and the He I $\lambda 5876$ and $\mathrm{H} \alpha$ lines in the red channel. The operation was performed separately for the MODS1 and MODS2 spectra, for a total of four velocity estimates for each region. The velocity measurements turned out to be more accurate (as quantified by the standard deviation) for the red channel than for the blue one, and for MODS2 than for MODS1. The red and blue channel velocities typically agreed within the uncertainties.

The final velocities were then computed as the weighted mean of the four different values, and we adopted the weighted standard deviation as our velocity uncertainty. The derived velocities were transformed into heliocentric ones using the rvcorrect task in IRAF. The results are provided at the end of Table 2. The velocities derived for our six H II regions range from $479 \pm 1 \mathrm{~km} / \mathrm{s}$ to $522 \pm 1 \mathrm{~km} / \mathrm{s}$. For comparison, systemic velocities for DDO 68 derived in the literature from neutral and ionized gas are in the range $V_{\text {sys }} \sim 500-510 \mathrm{~km} / \mathrm{s}$ (Ekta, Chengalur \& Pustilnik 2008; Cannon et al. 2014; Moiseev 2014).

It is useful to compare our results with the velocity map of the ionized gas derived by Moiseev (2014) for DDO 68 (see their Fig. 1). Moiseev (2014) found a velocity gradient in the north-south direction, with $V-V_{\text {sys }}$ ranging from $\sim-40 \mathrm{~km} / \mathrm{s}$ to $\sim+40 \mathrm{~km} / \mathrm{s}$ from the "tail" to the "head" of the comet. This behaviour is consistent with the rotation observed in the HI (Ekta, Chengalur \& Pustilnik 2008; Cannon et al. 2014), and indicates that neutral and ionized gas rotate in the same direction. Our velocity measurements are consistent with this trend since the most external Reg. 7 exhibits the lowest radial velocity $(479 \pm 1 \mathrm{~km} / \mathrm{s})$, whereas the highest velocity $(522 \pm 1 \mathrm{~km} / \mathrm{s})$ is found in Reg. 3 in the head of the comet.
For comparison, the HI velocities as inferred from the velocity contours displayed in Fig. 2 of Cannon et al. (2014) are $\sim 480 \mathrm{~km} / \mathrm{s}$ for Reg. 7 and $\sim 530 \mathrm{~km} / \mathrm{s}$ for Reg. 1 and 3 , consistent with our values. For Reg. 4 , the HI velocity is $\sim 525 \mathrm{~km} / \mathrm{s}$, while we find a lower velocity of $508 \pm 4 \mathrm{~km} / \mathrm{s}$. For Reg. 9, the HI velocity is $\sim 510 \mathrm{~km} / \mathrm{s}$ versus our Hil region value of $519 \pm 7 \mathrm{~km} / \mathrm{s}$, while for Reg. 8 , the innermost one, the HI velocity is $\sim 505 \mathrm{~km} / \mathrm{s}$ versus our lower value of $487 \pm 6$ $\mathrm{km} / \mathrm{s}$. Therefore we conclude that, on a first approximation, the ionized gas kinematics trace the behaviour of the HI; small deviations from the HI behaviour are nonetheless present, which is not surprising since $\mathrm{H}$ II region peculiar velocities, caused by e.g. expanding shells, can significantly distort the disk velocity field due to the relatively small amplitude of DDO 68 's rotation curve $\left(V_{c}=45 \mathrm{~km} / \mathrm{s}\right.$ at $11 \mathrm{kpc}$ radius, Cannon et al. 2014). Indeed, Pustilnik et al. (2017) suggested that the arrangement of $\mathrm{H}$ II regions in a ring-like structure in the head of the comet is due to the feedback effect from a supergiant shell expanding at a velocity of $\sim 13$ $\mathrm{km} / \mathrm{s}$.

As mentioned in the introduction, Annibali et al. (2016) presented an N-body simulation of three interacting galaxies that, under a given line of sight, is able to reproduce the morphology of the tail, the stream and the arc in DDO 68. With the new information from our derived radial velocities, combined with literature studies of the ionized and HI gas, we will be able to perform new better-constrained dynamical simulations of the DDO 68 system.

\section{DISCUSSION AND CONCLUSIONS}

In this paper we have presented LBT/MODS spectroscopy of six $\mathrm{H}$ II regions in DDO 68, two candidate companion galaxies, and one candidate star cluster. Our target $\mathrm{H}$ II regions cover all the main components of the DDO 68 system (namely: main body, cometary tail and northern ring), except the small stream discovered by Annibali et al. (2016), whose objects are too faint for spectroscopy even with the LBT. Both the galaxies and the candidate cluster turned out to be background galaxies.

We confirm the extremely low metallicity of DDO 68 and furthermore provide the first direct-method abundance measurement in the "tail" (Reg. 7); here we derive a metallicity as low as $12+\log (\mathrm{O} / \mathrm{H})=6.96 \pm 0.09$, potentially lower, within the uncertainties, than that of the record-holder galaxy J0811+4730, whose oxygen abundance is $12+\log (\mathrm{O} / \mathrm{H})=6.98 \pm 0.02$ (Izotov et al. 2018).

As a consequence, we confirm that DDO 68 is an extreme outlier in the luminosity-metallicity relation. Figure 8 shows the relation as inferred from the data by various authors and our DDO 68 values for Reg. 1, 3 and 7 , all obtained with the direct $\mathrm{T}_{e}$ method; DDO 68's absolute $\mathrm{B}$ magnitude is $M_{B}=-15.9$, computed adopting $\mathrm{B}=14.69$ (Taylor et al. 2005), a foreground extinction of $\mathrm{A}_{B}=0.08$ (Schlegel et al. 1998), and a distance modulus of $(m-M)_{0}=30.52$ (Sacchi et al. 2016). The average oxygen abundance inferred for DDO 68' s H II regions is a factor of $\sim$ ten lower than expected for its luminosity. We also show in the figure a hypothetical central abundance for DDO 68 obtained by extrapolating the linear fit in Fig. 5 to $\mathrm{R}=0$; even this value is significantly below the luminosity- 


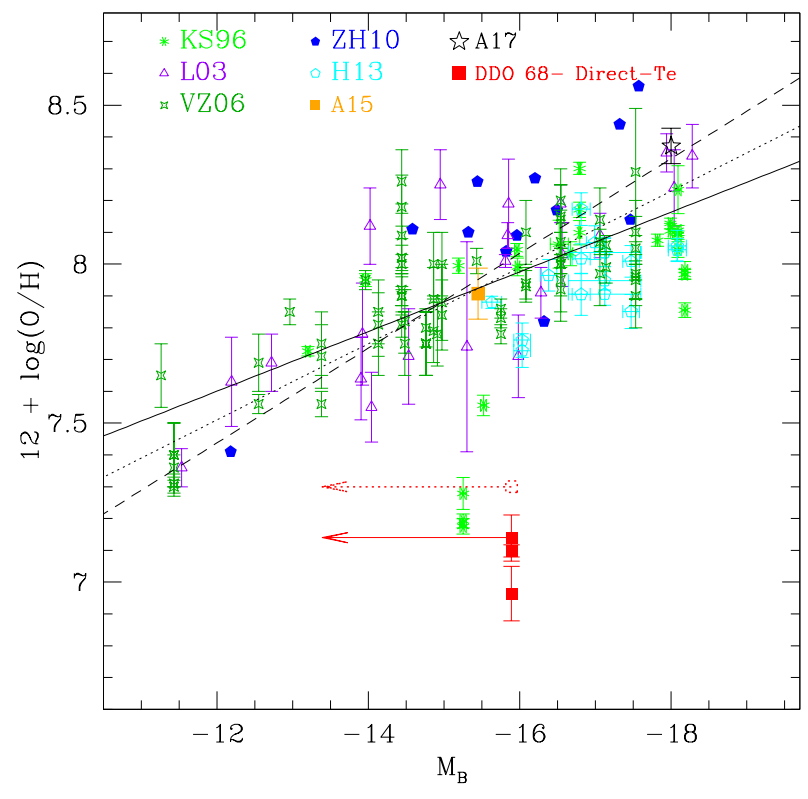

Figure 8. Luminosity - metallicity (L-Z) relation in star-forming dwarfs from the literature. All abundances have been obtained with the direct $\mathrm{T}_{\boldsymbol{e}}$ method. Red filled squares indicate the abundances derived by us for Reg.1, 3 and 7 in DDO 68 with the direct $\mathrm{T}_{e}$ method. The dotted square at $12+\log (O / H) \sim 7.3$ is the value extrapolated at $\mathrm{R}=0$ from the linear fit in Fig. 5 . The arrows indicate the position of DDO 68 in the L-Z relation assuming that the measured H II regions originated from a secondary acquired body with mass $1 / 10$ that of DDO 68 . The other symbols correspond to the labelled references: KS -Kobulnicky \& Skillman (1996), L03 - Lee et al. (2003), VZ06 - van Zee \& Haynes (2006), ZH10 - Zhao et al. (2010), H13 - Haurberg et al. (2013), A15 Annibali et al. (2015), A17 - Annibali et al. (2017). The solid line is the $3-\sigma$ rejection linear least squares fit to the data points, excluding DDO 68: $12+\log (\mathrm{OH})=(-0.094 \pm 0.001) \times M_{B}+(6.47 \pm 0.12)$. The dashed and dotted lines are, respectively, the relations from van Zee \& Haynes (2006) and Haurberg et al. (2013).

metallicity relation, by a factor of about 5 . If we attributed the $\mathrm{H}$ II regions to a secondary acquired body with mass, and hence luminosity, ten times lower that DDO 68, and thus shifted them to the left, they would still remain outside the plot area occupied by the other galaxies (see arrows in Fig. 8), with only the extrapolated central value being marginally consistent with the relation.

From synthetic CMDs, Sacchi et al. (2016) derived a total stellar mass of $\sim 10^{8} M_{\odot}$ for DDO 68 , which allows us to explore the position of this galaxy on the mass-metallicity (M-Z) relation; taking as reference the distribution of galaxies in Fig. 8 of Berg et al. (2012), we conclude that DDO 68 is an outlier also in the M-Z relation. It is worth recalling that the other known XMPs do not deviate from the overall relations. Also Leo P (Skillman et al. 2013), which is somewhat similar to DDO 68 for what concerns the low metallicity and the high $\mathrm{N} / \mathrm{O}$, is perfectly aligned with the other dwarfs (Berg et al. 2012), thanks to its much lower luminosity (and mass).

The overall interpretation of the luminosity - metallicity relation is that late-type dwarfs with higher gas fractions and lower masses are more inefficient at chemically enrich- ing their medium, both because they have proportionally larger amounts of gas to pollute and because they have more chances to lose their metals through galactic winds, due to their lower potential well (e.g., Chisholm et al. 2018). This scenario can still apply also to DDO 68, but the question is why has its enrichment been so less efficient than the others. How did DDO 68 components manage to get rid of most of the oxygen produced by their stars? Or to dilute it with exceptional amounts of primordial external gas? In the past, some (Izotov \& Thuan 1999) suggested that XMPs are experiencing now their first burst of star formation, and have not had time to significantly enrich and mix their medium. However, we now know that this is not the case for any of the dwarfs studied with deep HST photometry in the local Universe (see, e.g., Tolstoy, Hill \& Tosi 2009; McQuinn et al. 2010; Weisz et al. 2011, and references therein), all of which clearly contain stars as old as the lookback time reachable at their distance. In particular, DDO 68, as well as IZw18 and all the other XMPs within $\sim 18 \mathrm{Mpc}$, hosts also RGB stars, i.e. stars at least 2-3 Gyr, possibly 13 Gyr old (Aloisi et al. 2007; Sacchi et al. 2016). The SFHs derived from our HST photometry by Sacchi et al. (2016) and, independently, by Makarov et al. (2017) agree that the system has been forming stars since the oldest epochs, although at a quite low rate until a few hundred Myr ago; hence oxygen must have been synthesized.

Thanks to the space distribution of our targets, we have been able to detect a dependence of the oxygen abundance on the distance from the galaxy center, whose linear fit slope $\Delta \log (\mathrm{O} / \mathrm{H}) / \Delta \mathrm{R}$ is in the range -0.06 to $-0.12 \mathrm{dex} \mathrm{kpc}^{-1}$, or -0.30 to -0.59 dex $R_{25}^{-1}$, with the flatter values from the direct $\mathrm{T}_{\boldsymbol{e}}$ method, and the steepest ones from the indirect one. Unfortunately, the sample is too small to assess whether this dependence is actually a metallicity gradient similar to those observed in all spiral galaxies (see, e.g., Wilson \& Rood 1994; Sanchez et al. 2014, and references therein) and, recently, in some star-forming dwarfs (Annibali et al. 2015; Pilyugin et al. 2015; Annibali et al. 2017), or the result of a yet incomplete mixing of DDO 68's two merging bodies, the main body and its satellite cometary tail (with or without the head in the northern ring). A combination of the two explanations is also possible, since born-in-situ gradients are the consequence of the spatial dependence of the ratio between star formation history (i.e. element production, usually higher in the inner regions) and infall of metalpoor gas (i.e. element dilution, usually higher in the outer regions), with the possible complication of galactic outflows (element loss, where the potential well is sufficiently low). At any rate, the $\mathrm{H}$ II regions in the tail have oxygen abundances lower than most of the others, hence the oxygen gradient observed in DDO 68 may be the consequence of the accretion of both metal-poor gas and the metal-poorer satellite.

In a forthcoming paper (Romano et al. in preparation), we will address the evolution of the system with chemical evolution models taking into account the known $\mathrm{SFH}$, the possible interaction histories of the system and the N-body simulations presented by Annibali et al. (2016), to try to disentangle whether the anomalously low observed oxygen is more likely due to the interactions, to unusually high accretion of primordial gas, or to galactic winds. We will furthermore investigate the reason for the high N/O observed in DDO 68, trying to disentangle whether it can 
be explained with nitrogen enrichment from massive, lowmetallicity rotating stars, as predicted by stellar models (e.g. Maeder \& Meynet 2001; Meynet \& Maeder 2002), or if the reason is the age of the strongest burst occurred in DDO 68 (from $\sim 10 \mathrm{Myr}$ to $\sim 300 \mathrm{Myr}$ ago), combined with the delayed production of nitrogen with respect to oxygen (Henry et al. 2000).

Finally, we measured radial velocities in the range $479 \pm 1$ $\mathrm{km} / \mathrm{s}$ to $522 \pm 1 \mathrm{~km} / \mathrm{s}$ from the tail to the head of the "comet" in DDO 68, consistent with the rotation derived in the $\mathrm{H} \mathrm{I}$ by Cannon et al. (2014).

\section{ACKNOWLEDGMENTS}

We thank the anonymous referee for his/her highly valuable comments and suggestions and for his/her very accurate report, which contributed to significantly improve the paper. F.A., D.R. and M.T. kindly acknowledge funding from INAF PRIN-SKA-2017 program 1.05.01.88.04. This work was based on LBT/MODS data. The LBT is an international collaboration among institutions in the United States, Italy and Germany. LBT Corporation partners are: the University of Arizona on behalf of the Arizona Board of Regents; Istituto Nazionale di Astrofisica, Italy; LBT Beteiligungsgesellschaft, Germany, representing the Max-Planck Society, the Leibniz Institute for Astrophysics Potsdam, and Heidelberg University; the Ohio State University, and the Research Corporation, on behalf of the University of Notre Dame, University of Minnesota and University of Virginia.

\section{APPENDIX A: LBT/MODS SPECTRA OF TARGET REGIONS.}

The LBT/MODS spectra of $\mathrm{H}$ II regions 3, 4, 7, 8 and 9 in DDO 68 , of the two candidate companion galaxies (Gal. 1 and Gal. 2), and of the candidate star cluster (CL) are shown in Figures A1 to A8.

\section{APPENDIX B: "RAW" EMISSION LINE FLUXES}

We provide in Tables B1 the emission line fluxes, with no reddening correction applied, for the six $\mathrm{H}$ II regions studied in DDO 68. The reported fluxes were obtained by averaging the results from MODS1 and MODS2, as outlined in Section 3, with the associated uncertainties computed as the standard deviation of the two different measurements.

\section{REFERENCES}

Aloisi, A., Clementini, G., Tosi, M., Annibali, F., Contreras, R., Fiorentino, G., Mack, J., Marconi, M., et al., 2007, ApJ, 667, L151

Annibali, F., Tosi, M., Pasquali, A., et al. 2015, AJ, 150, 143

Annibali, F., Nipoti, C., Ciotti, L., et al. 2016, ApJ, 826, L27

Annibali, F., Tosi, M., Romano, D., Buzzoni, A., Cusano, F., Fumana, M., Marchetti, A., Mignoli, M., et al. 2017, ApJ, 843,20

Aver, E., Olive, K. A., \& Skillman, E. D. 2011, J. Cosmology Astropart. Phys., 3, 043

Aver, E., Olive, K. A., Porter, R. L., \& Skillman, E. D. 2013, J. Cosmology Astropart. Phys., 11, 017

Berg, D. A., Skillman, E. D., Marble, A. R., van Zee, L., Engelbracht, C.W., Lee, J.C., Kennicutt, R.C., Jr., Calzetti, D., et al. 2012, ApJ, 754, 98

Berg, D. A., Skillman, E. D., Croxall, K. V., et al. 2015, ApJ, 806,16

Bresolin, F., Gieren, W., Kudritzki, R.-P., et al. 2009a, ApJ, 700, 309

Caffau, E., Ludwig, H.-G., Steffen, M., et al. 2008, A\&A, 488, 1031

Cannon, J. M., Johnson, M., McQuinn, K. B. W., et al. 2014, ApJ, 787, L1

Clegg, R. E. S. 1987, MNRAS, 229, 31P

Cardelli, J. A., Clayton, G. C., \& Mathis, J. S. 1989, ApJ, 345, 245

Chisholm, J., Tremonti, C., \& Leitherer, C. 2018, MNRAS, 481, 1690

D'Ercole, A., \& Brighenti, F. 1999, MNRAS, 309, 941

de Vaucouleurs, G., de Vaucouleurs, A., Corwin, H. G., Jr., et al. 1991, Third Reference Catalogue of Bright Galaxies. Volume I: . Springer, New York, NY (USA), 1991

Diemand, J., Kuhlen, M., Madau, P., et al. 2008, Nature, 454, 735

Diaz, A. I., \& Tosi, M. 1986, A\&A, 158, 60

Ekta, Chengalur, J.N., \& Pustilnik, S.A., 2008, MNRAS, 391, 881

Esteban, C., García-Rojas, J., Carigi, L., et al. 2014, MNRAS, 443,624

Fernández, V., Terlevich, E., Díaz, A. I., Terlevich, R., \& RosalesOrtega, F. F. 2018, arXiv:1804.10701

Filippenko, A. V. 1982, PASP, 94, 715

Garnett, D. R. 1990, ApJ, 363, 142

Garnett, D. R. 1992, AJ, 103, 1330

Gavilàn, M., Ascasibar, Y., Mollà, M., Díaz, A.I. 2013, MNRAS, 434,2491

Guseva, N. G., Izotov, Y. I., Stasińska, G., et al. 2011, A\&A, 529, A149

Hanuschik, R. W. 2003, A\&A, 407, 1157

Haurberg, N. C., Rosenberg, J., \& Salzer, J. J. 2013, ApJ, 765, 66

Hinshaw, G., Larson, D., Komatsu, E., et al. 2013, ApJS, 208, 19

Henry, R. B. C., Edmunds, M. G., \& Köppen, J. 2000, ApJ, 541, 660

Izotov, Y. I., \& Thuan, T. X. 1999, ApJ, 511, 639

Izotov, Y. I., Stasińska, G., Meynet, G., Guseva, N. G., \& Thuan, T. X. 2006, A\&A, 448, 955

Izotov, Y. I., Thuan, T. X., \& Stasińska, G. 2007b, ApJ, 662, 15

Izotov, Y. I., \& Thuan, T. X. 2007a, ApJ, 665, 1115

Izotov, Y. I. \& Thuan, T. X. 2009, ApJ, 690, 1797

Izotov, Y. I., Guseva, N. G., Fricke, K. J., \& Papaderos, P. 2009, A\&A, 503, 61

Izotov, Y. I., Thuan, T. X., Guseva, N. G., 2014, MNRAS, 445, 778

Izotov, Y. I., Thuan, T. X., Guseva, N. G., \& Liss, S. E. 2018, MNRAS, 473, 1956

James, B. L., Tsamis, Y. G., Walsh, J. R., Barlow, M. J., \& 


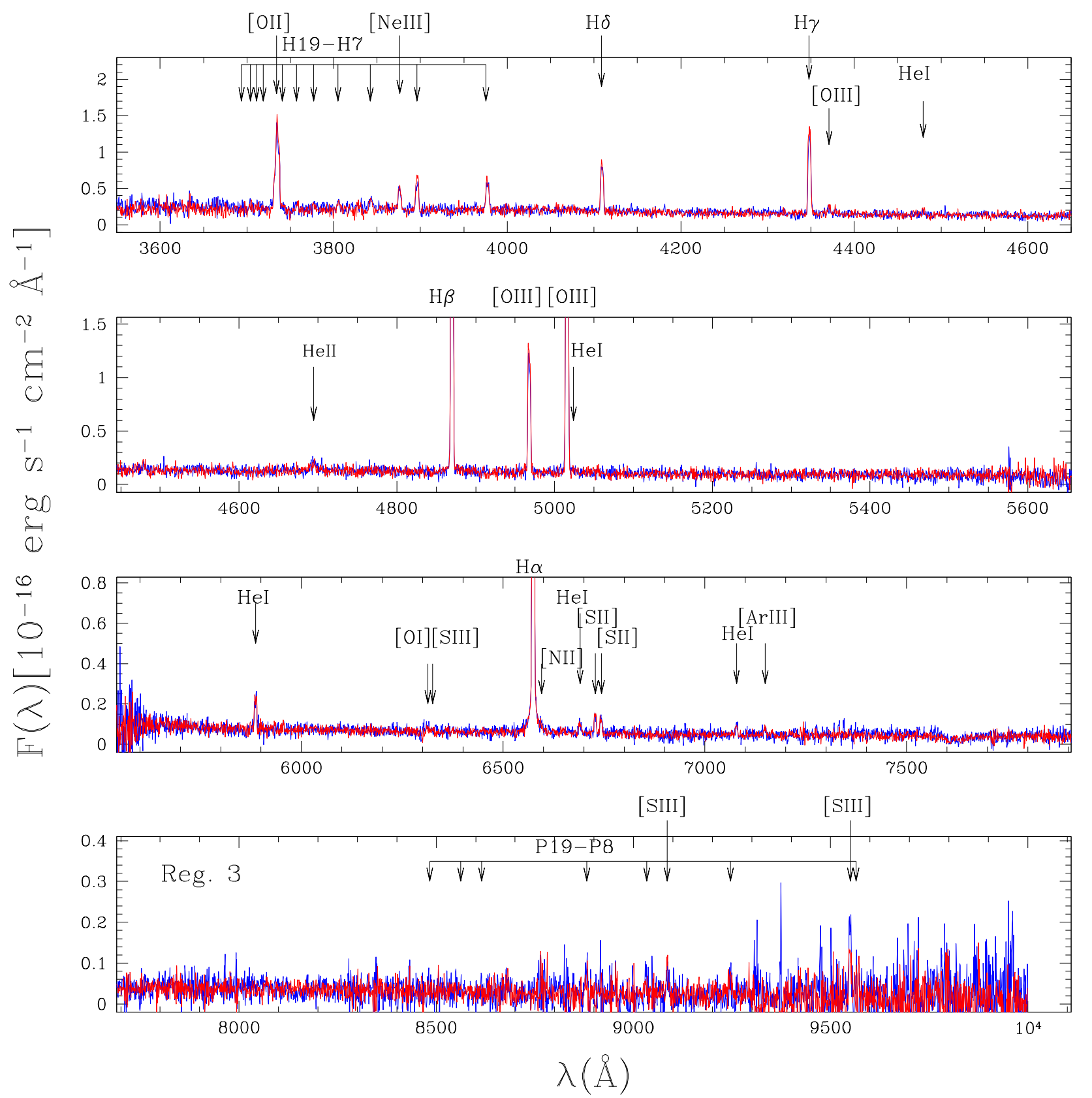

Figure A1. LBT/MODS spectra for Reg. 3 in DDO 68 acquired with MODS1 (blue line) and MODS2 (red line). Indicated are the most relevant emission lines.

Westmoquette, M. S. 2013, MNRAS, 448, 2687

Kennicutt, R. C., Jr., Bresolin, F., \& Garnett, D. R. 2003, ApJ, 591,801

Kewley, L. J., \& Ellison, S. L. 2008, ApJ, 681, 1183

Kobulnicky, H. A., \& Skillman, E. D. 1996, ApJ, 471, 211

Lee, H., McCall, M. L., Kingsburgh, R. L., Ross, R., \& Stevenson, C. C. 2003, AJ, 125, 146

Legrand, F., Kunth, D., Roy, J.-R., Mas-Hesse, J. M., \& Walsh, J. R. 2000, A\&A, 355, 891

Leitherer, C., Ekström, S., Meynet, G., et al. 2014, ApJS, 212, 14 Lequeux, J., Peimbert, M., Rayo, J.F., Serrano, A., TorresPeimbert, S. 1979, A\&A, 80, 155

Luridiana, V., Morisset, C., \& Shaw, R. A. 2015, A\&A, 573, A42
Mac Low, M.-M., \& Ferrara, A. 1999, ApJ, 513, 142

Maeder, A. \& Meynet, G. 2001, A\&A, 373, 555

Makarov, D.I., Makarova, L.N., Pustilnik, S.A., Borisov, S.B., 2017, MNRAS, 466, 556

Marconi, G., Matteucci, F., \& Tosi, M. 1994, MNRAS, 270, 35

Matteucci, F., \& Tosi, M. 1985, MNRAS, 217, 391

McQuinn, K. B. W., Skillman, E. D., Cannon, J. M., et al. 2010, ApJ, 724, 49

Meynet, G., \& Maeder, A. 2002, A\&A, 390, 561

Moiseev, A. V. 2014, Astrophysical Bulletin, 69, 1

Nava, A., Casebeer, D., Henry, R. B. C., \& Jevremovic, D. 2006, ApJ, 645, 1076

Olive, K. A., \& Skillman, E. D. 2004, ApJ, 617, 29 


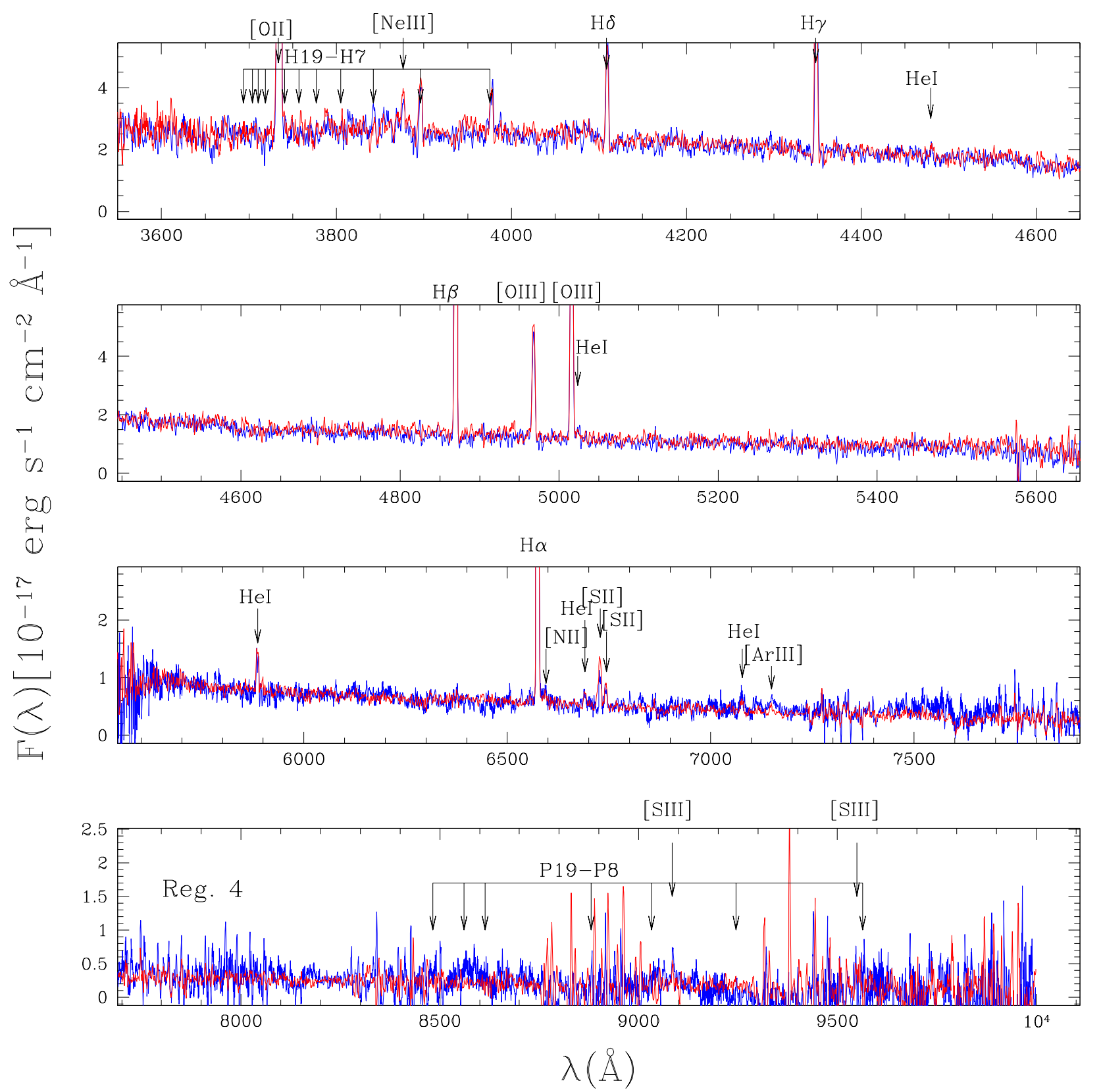

Figure A2. LBT/MODS spectra for Reg. 4 in DDO 68 acquired with MODS1 (blue line) and MODS2 (red line). Indicated are the most relevant emission lines.

Osterbrock, D. E. 1989, Research supported by the University of California, John Simon Guggenheim Memorial Foundation, University of Minnesota, et al. Mill Valley, CA, University Science Books, 1989, 422 p.,

Pagel, B. E. J. 1985, European Southern Observatory Conference and Workshop Proceedings, 21, 155

Pagel, B. E. J., Simonson, E. A., Terlevich, R. J., \& Edmunds, M. G. 1992, MNRAS, 255, 325

Peimbert, M., Luridiana, V., \& Peimbert, A. 2007, ApJ, 666, 636 Peimbert, M., \& Torres-Peimbert, S., 1974, ApJ, 193, 327

Pilyugin, L. S. 1992, A\&A, 260, 58

Pilyugin, L. S., \& Thuan, T. X. 2005, ApJ, 631, 231

Pilyugin, L. S., Grebel, E. K., \& Mattsson, L. 2012, MNRAS, 424,
2316

Pilyugin, L. S., Grebel, E. K., \& Zinchenko, I. A. 2015, MNRAS, 450,3254

Pilyugin, L. S., \& Grebel, E. K. 2016, MNRAS, 457, 3678

Planck Collaboration, Ade, P. A. R., Aghanim, N., et al. 2011, A\&A, 536, A1

Planck Collaboration: Ade, P. A. R., et al. 2016, A\&A, 594, A13 Porter, R. L., Ferland, G. J., Storey, P. J., \& Detisch, M. J. 2012, MNRAS, 425, L28

Porter, R. L., Ferland, G. J., Storey, P. J., \& Detisch, M. J. 2013, MNRAS, 433, L89

Pustilnik, S. A., Kniazev, A. Y., \& Pramskij, A. G. 2005, A\&A, 443, 91 


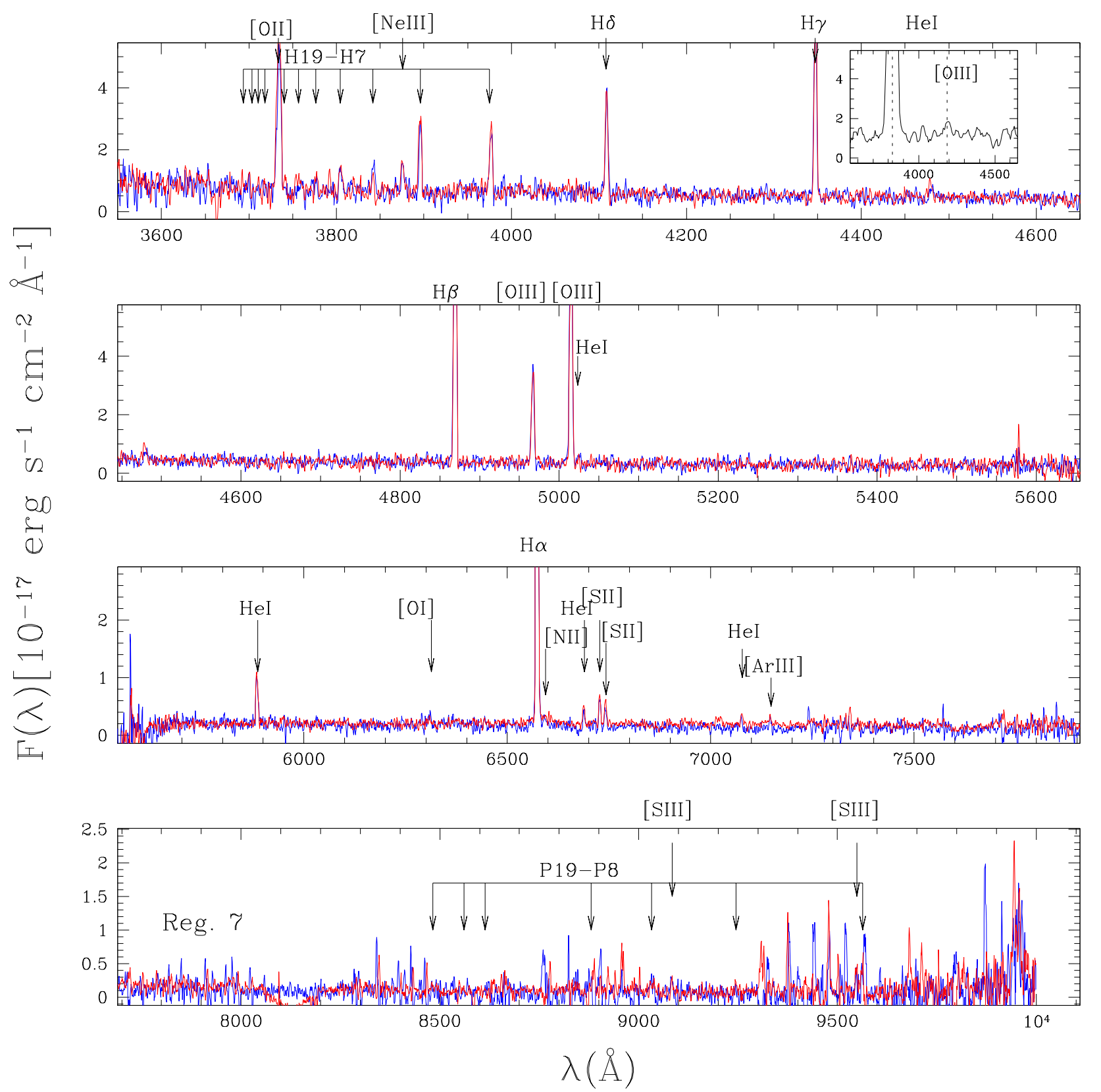

Figure A3. LBT/MODS spectra for Reg. 7 in DDO 68 acquired with MODS1 (blue line) and MODS2 (red line). Indicated are the most relevant emission lines. The small insertion in the top panel shows the spectrum obtained from the combination of all MODS1 and MODS2 data, where the $[\mathrm{O}$ III $] \lambda 4363$ detection is visible.

Pustilnik, S. A., Tepliakova, A. L., \& Kniazev, A. Y. 2007, arXiv:0712.4007

Pustilnik, S. A., Tepliakova, A. L., Kniazev, A. Y., \& Burenkov, A. N. 2008, MNRAS, 388, L24

Pustilnik, S. A., \& Tepliakova, A. L. , 2011, MNRAS, 415, 1188

Pustilnik, S. A., Makarova, L. N., Perepelitsyna, Y. A., Moiseev, A. V., Makarov, D. I., 2016, MNRAS, 465, 4985

Pustilnik, S. A., Makarova, L. N., Perepelitsyna, Y. A., Moiseev, A. V., \& Makarov, D. I. 2017, MNRAS, 465, 4985

Recchi, S., Spitoni, E., Matteucci, F., \& Lanfranchi, G. A. 2008, A\&A, 489, 555
Renzini, A., \& Voli, M. 1981, A\&A, 94, 175

Romano, D., Tosi, M., \& Matteucci, F. 2006, MNRAS, 365, 759

Romano, D., \& Starkernurg, E.. 2013, MNRAS, 434, 471

Sacchi, E., Annibali, F., Cignoni, M., et al. 2016, ApJ, 830, 3

Sanchez, S. F., Rosales-Ortega, F. F., Iglesias-Paramo, J., Molla, M., Barrera-Ballesteros, J., Marino, R. A., Perez, E., SanchezBlazquez, P., et al., 2014, A\&A, 563, 49

Scannapieco, E., \& Brüggen, M. 2010, MNRAS, 405, 1634

Schlegel, D. J., Finkbeiner, D. P., \& Davis, M. 1998, ApJ, 500, 525

Skillman, E.D., Kennicutt, R.C., Hodge, P.W. 1989, ApJ, 347, 

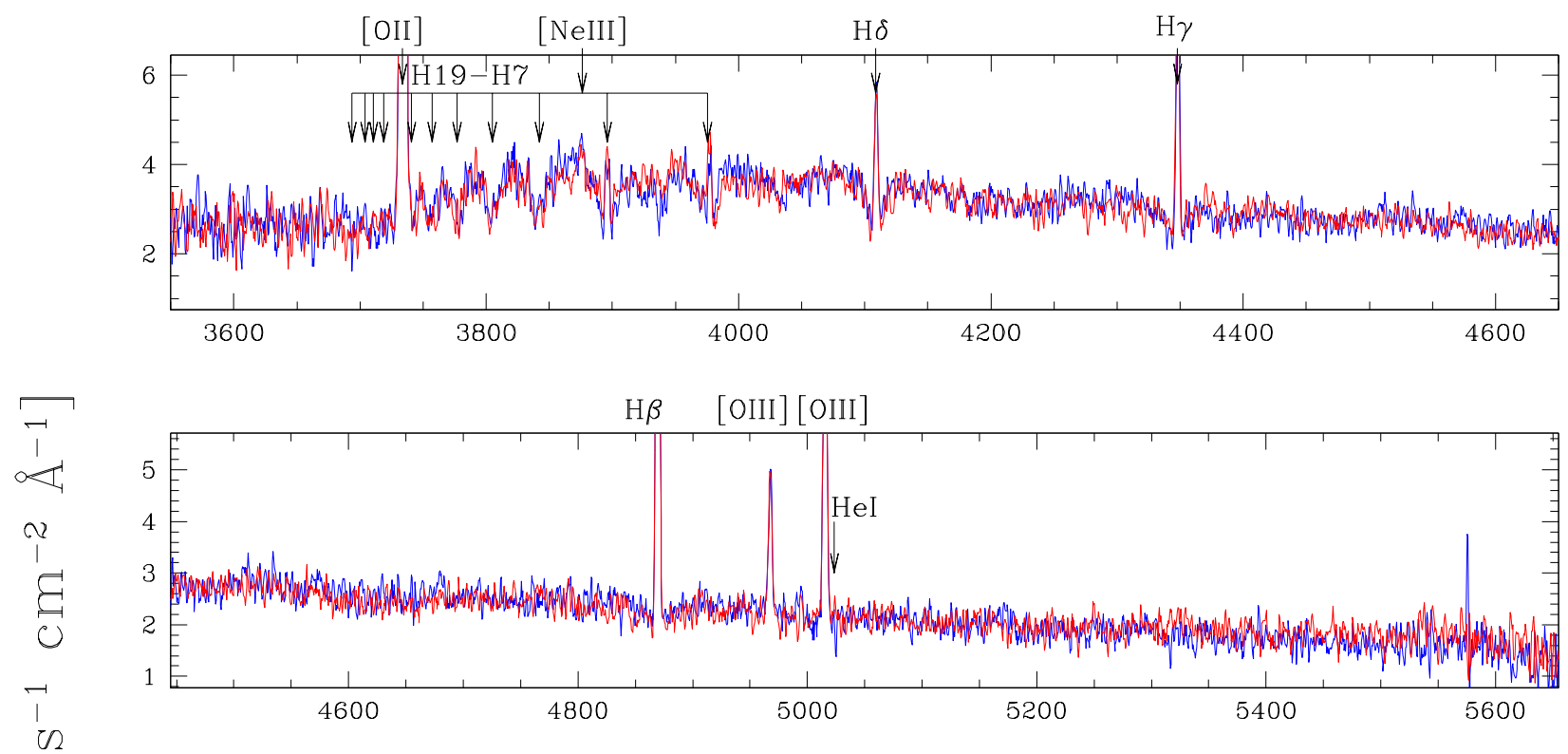

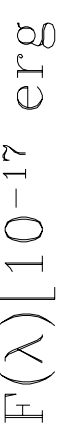

$\mathrm{H} \alpha$

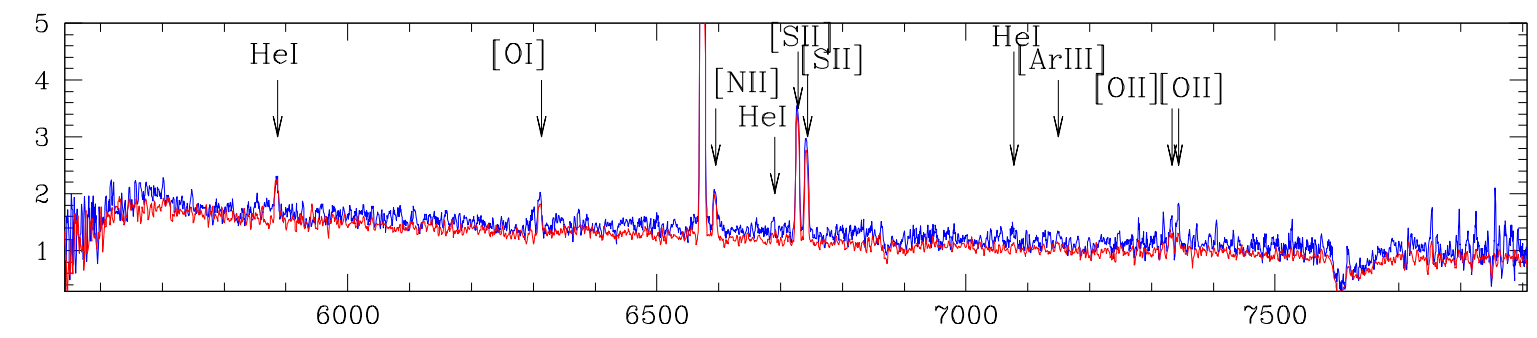

[SIII]

[SIII]

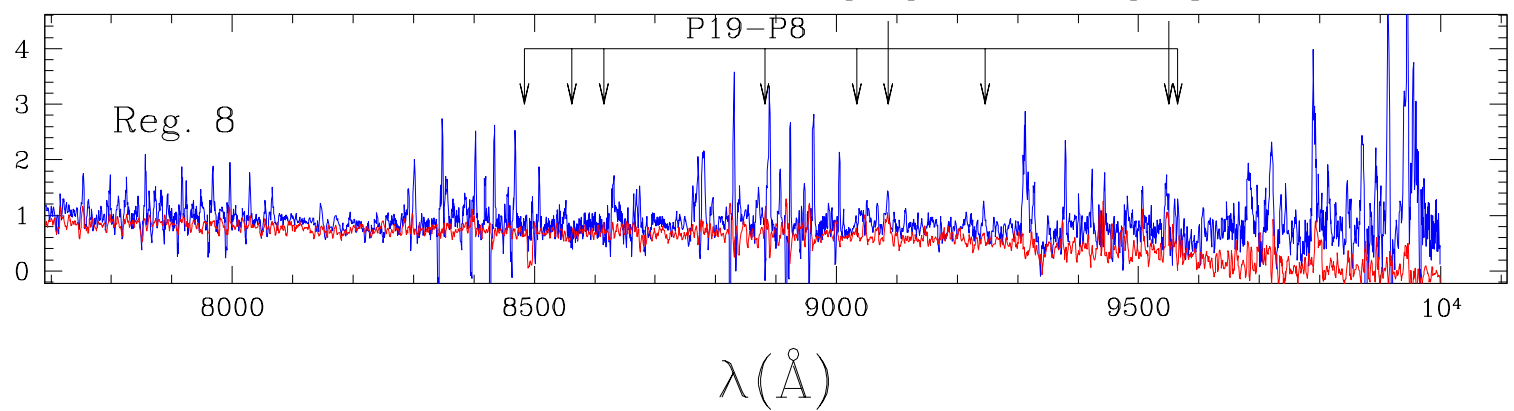

Figure A4. LBT/MODS spectra for Reg. 8 in DDO 68 acquired with MODS1 (blue line) and MODS2 (red line). Indicated are the most relevant emission lines.

875

Skillman, E.D., Salzer, J.J., Berg, D.A., Pogge, R.W., Haurberg,

N.C., Cannon, J.M., Aver, E., Olive, K.A. et al., 2013, AJ, 146,3

Spergel, D. N., Bean, R., Doré, O., et al. 2007, ApJS, 170, 377 Storey, P. J., \& Hummer, D. G. 1995, MNRAS, 272, 41

Spergel, D. N., Verde, L., Peiris, H. V., et al. 2003, ApJS, 148, 175

The Review of Particle Physics, J. Beringer et al., (Particle Data Group), 2012, Phys. Rev. D 86, 010001

Taylor, V. A., Jansen, R. A., Windhorst, R. A., Odewahn, S. C., \& Hibbard, J. E. 2005, ApJ, 630, 784

Tikhonov, N. A., Galazutdinova, O. A., \& Lebedev, V. S., 2014,
Astronomy Letters, 40, 1

Timmes, F.X., Woosley, S.E., \& Weaver, T.A., 1991, ApJS, 98, 617

Tinsley, B.M., 1980, Fund.Cosmic Phys. 5, 287

Tolstoy, E., Hill, V., \& Tosi, M. 2009, ARA\&A, 47, 371

van Zee, L., Salzer, J. J., \& Haynes, M. P. 1998, ApJ, 497, L1

van Zee, L., \& Haynes, M. P. 2006, ApJ, 636, 214

Vila-Costas, M.B., \& Edmunds, M.G. 1993, MNRAS, 265, 199

Weisz, D. R., Dalcanton, J. J., Williams, B. F., et al. 2011, ApJ, 739,5

Wheeler, C., Onorbe, J., Bullock, J.S., Boylan-Kolchin, M., Elbert, O.D., Garrison-Kimmel, S., Hopkins, P.F., Keres, D., 2015, MNRAS, 453, 1305 

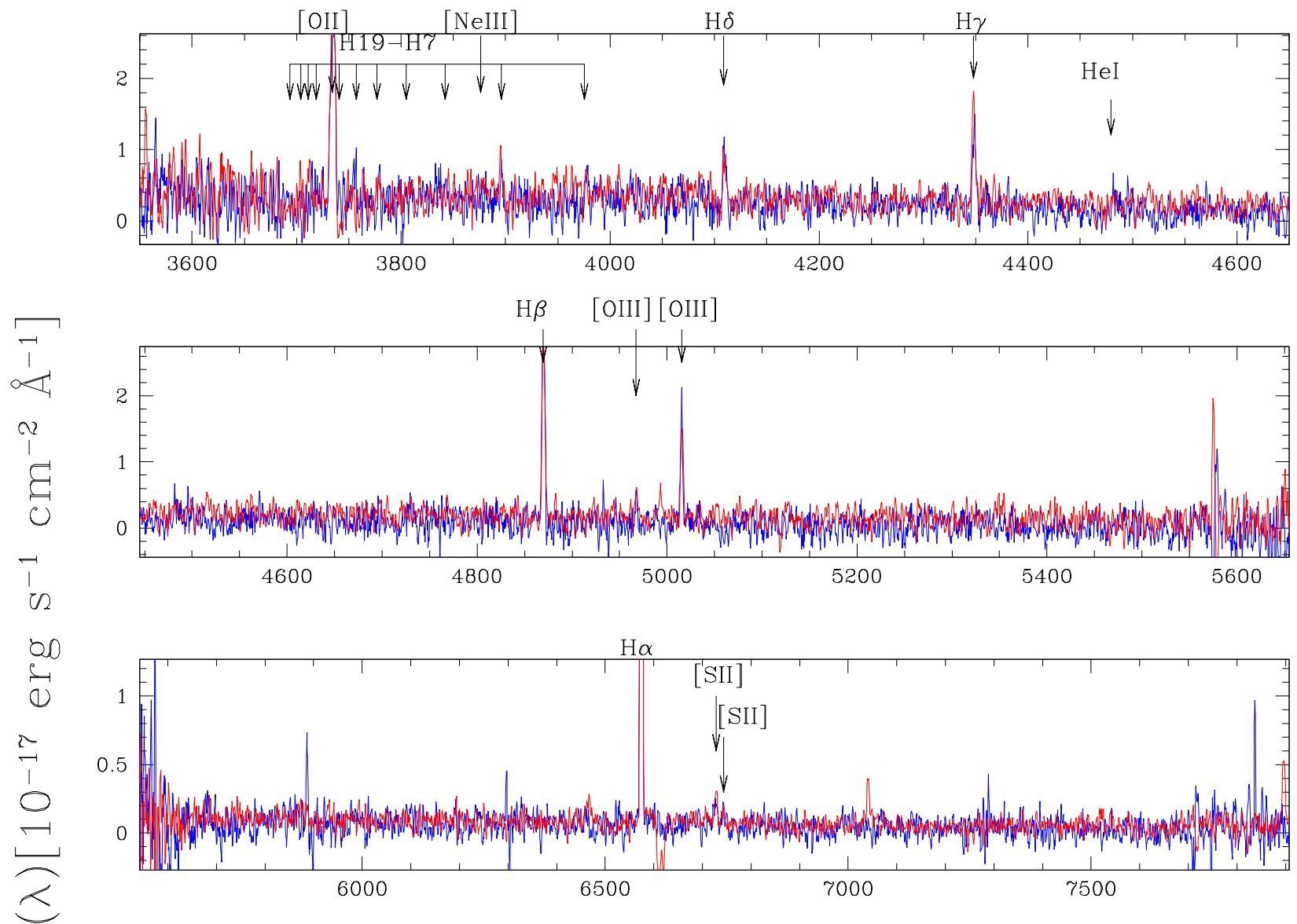

厌

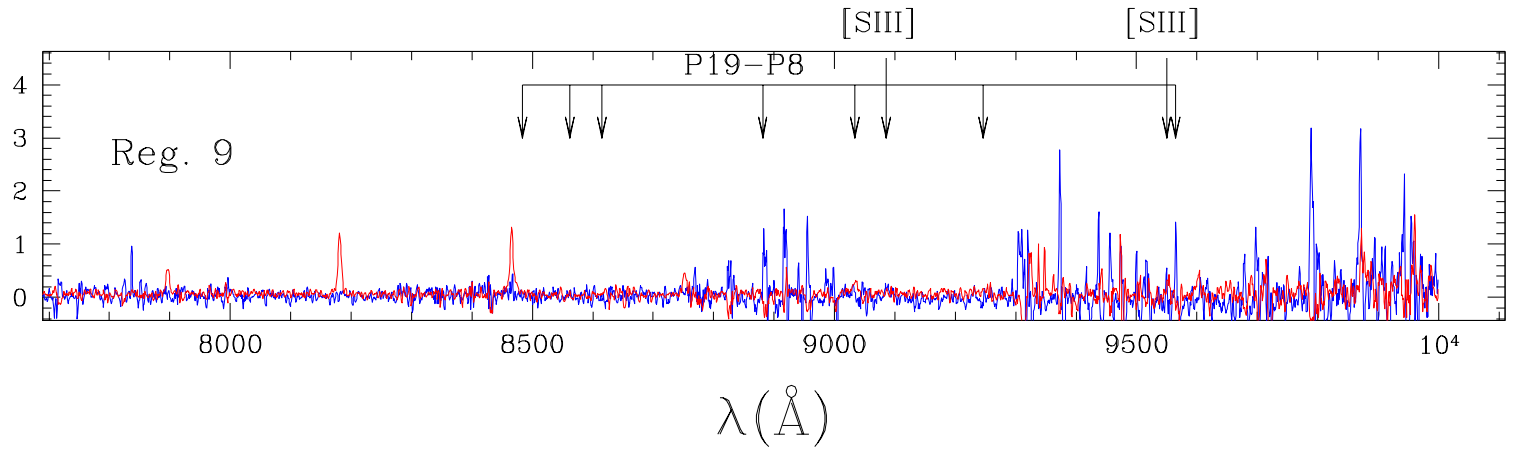

Figure A5. LBT/MODS spectra for Reg. 9 in DDO 68 acquired with MODS1 (blue line) and MODS2 (red line). Indicated are the most relevant emission lines.

Wilson, T.L., \& Rood, R., 1994, ARA\&A, 32, 191

Yang, J., Turner, M. S., Steigman, G., Schramm, D. N., Olive, K.

A., ApJ, 281, 493

Yin, S. Y., Liang, Y. C., Hammer, F., et al. 2007, A\&A, 462, 535

Zhao, Y., Gao, Y., \& Gu, Q., 2010, ApJ, 710, 663

Zinchenko, I. A., Pilyugin, L. S., Grebel, E. K., Sánchez, S. F., \&

Vílchez, J. M. 2016, MNRAS, 462, 2715

This paper has been typeset from a TEX/LATEX file prepared by the author. 

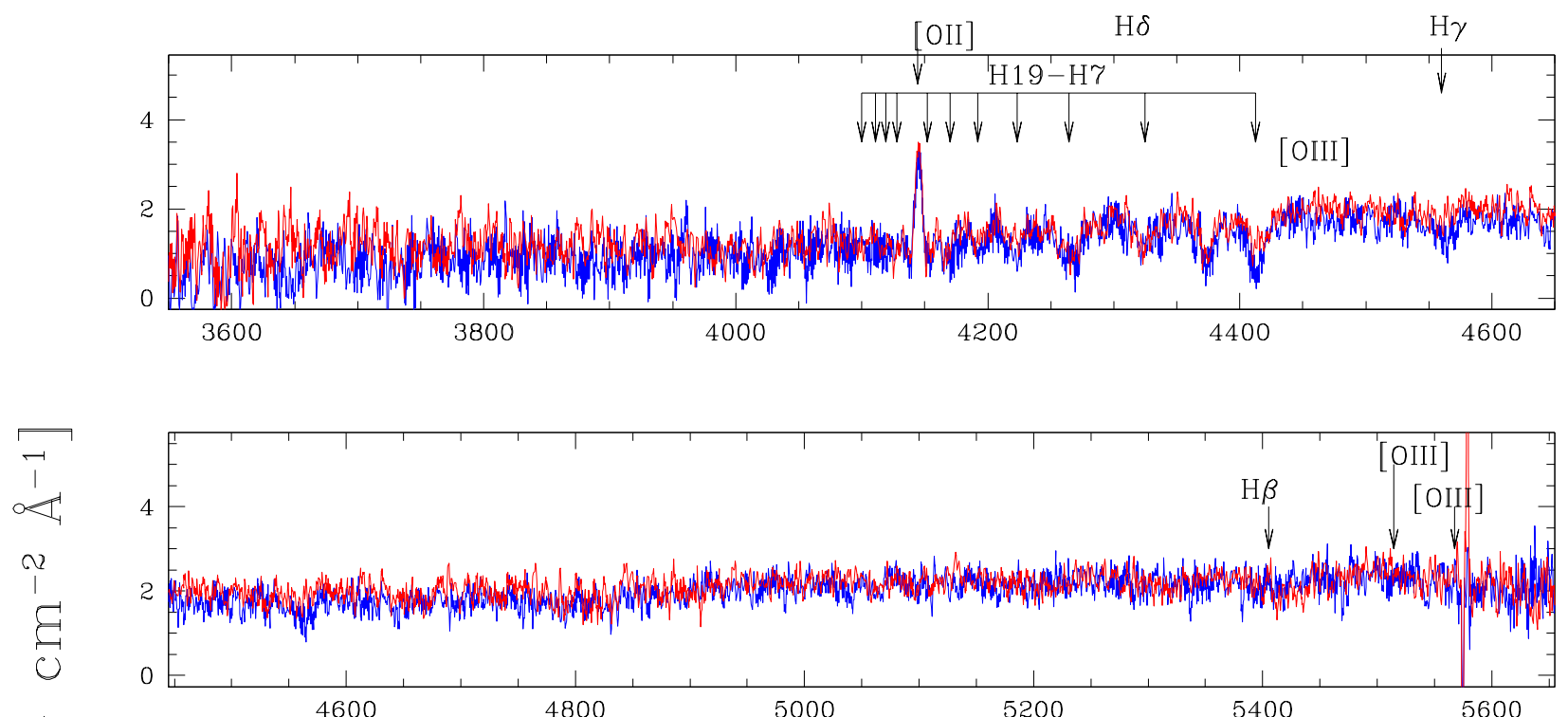

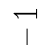
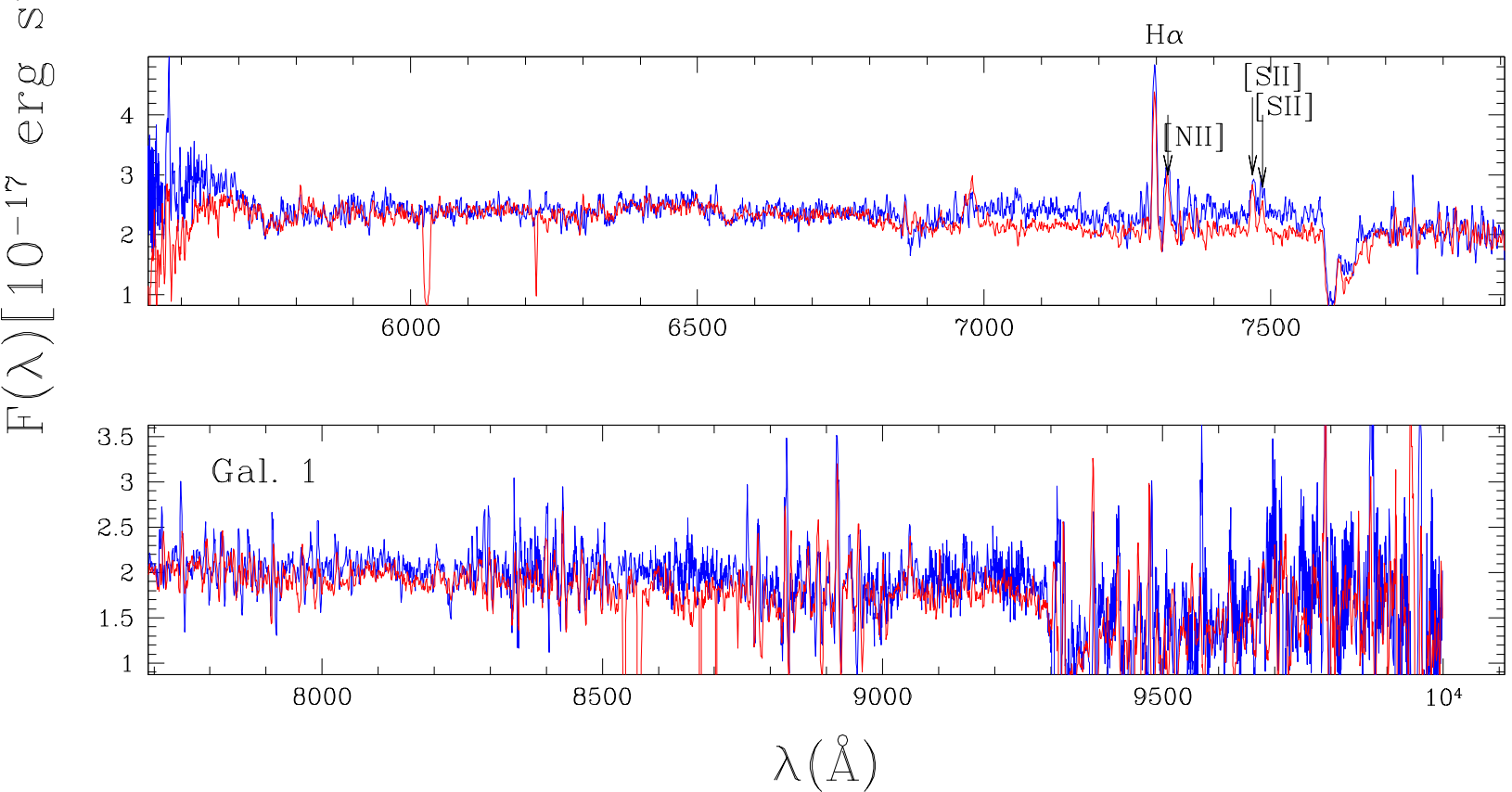

Figure A6. LBT/MODS spectra for Gal. 1, one of the two candidate companion galaxies of DO 68, acquired with MODS1 (blue line) and MODS2 (red line). Indicated are the most relevant emission and absorption lines. The system turned out to be an emission-line galaxy at $\mathrm{z} \sim 0.11$. 


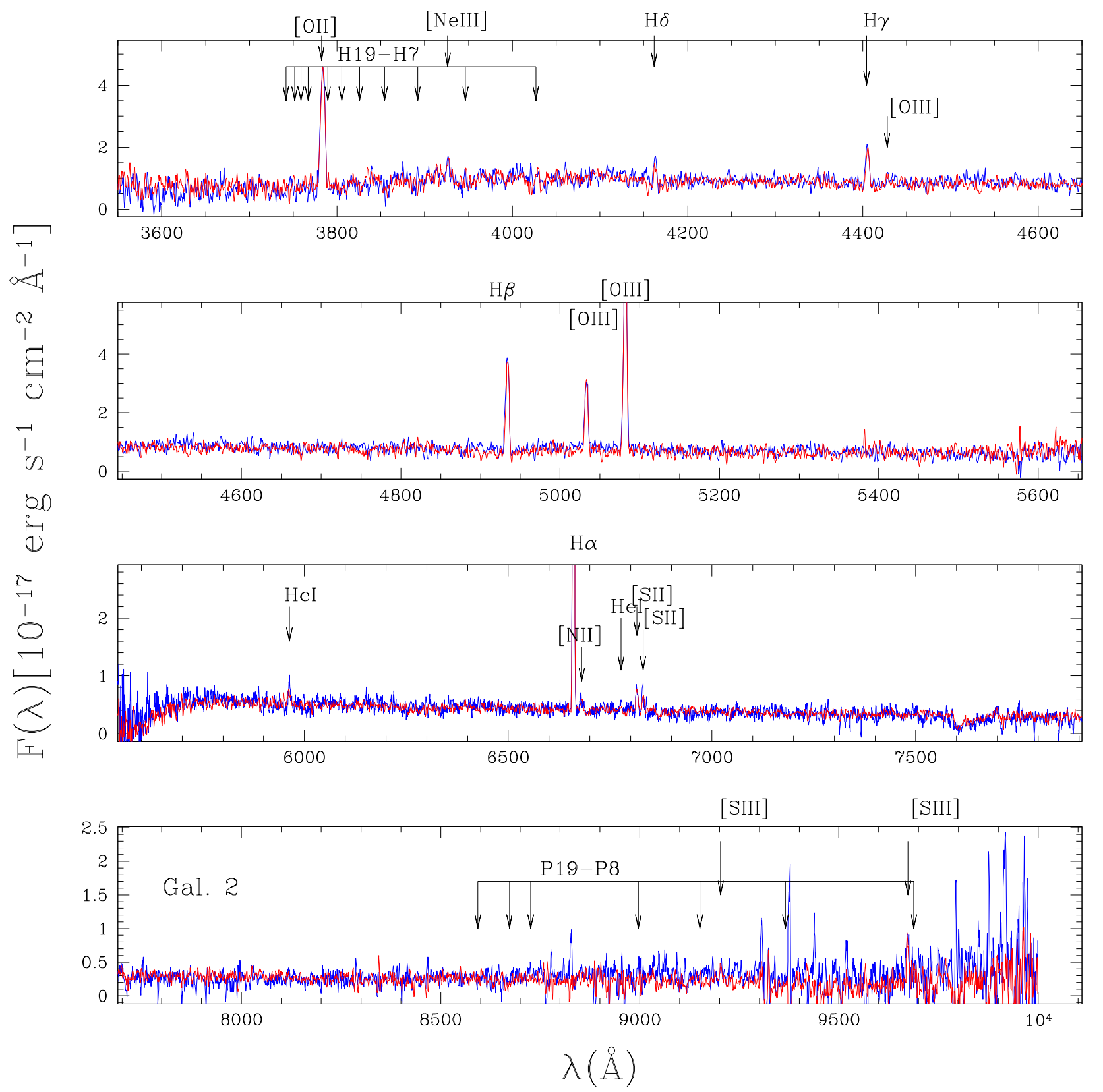

Figure A7. LBT/MODS spectra for Gal. 2, one of the two candidate companion galaxies of DO 68, acquired with MODS1 (blue line) and MODS2 (red line). Indicated are the most relevant emission lines. The system turned out to be an emission-line galaxy at $\mathrm{z} \sim 0.0148$. 

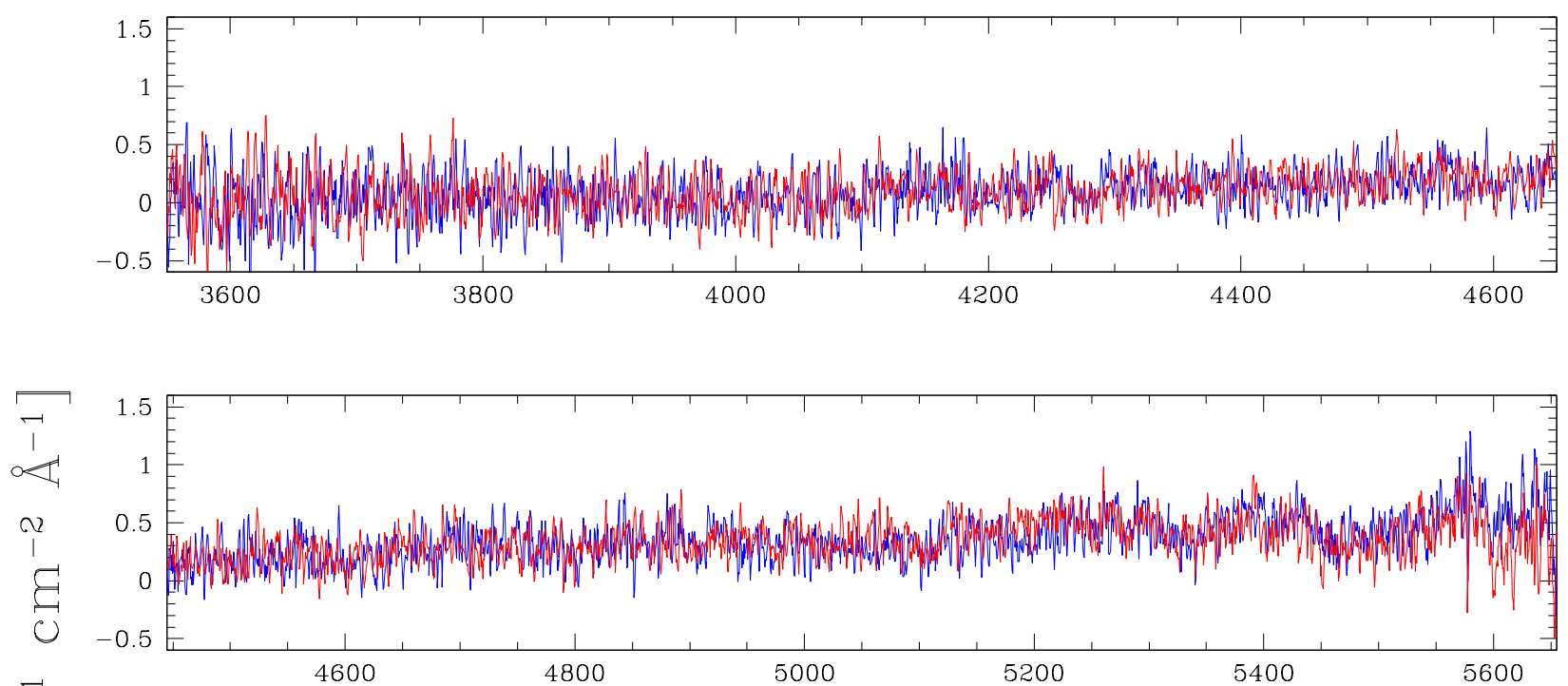

广

2
20
4
0
2
1
0
$E$
$E$
$E$
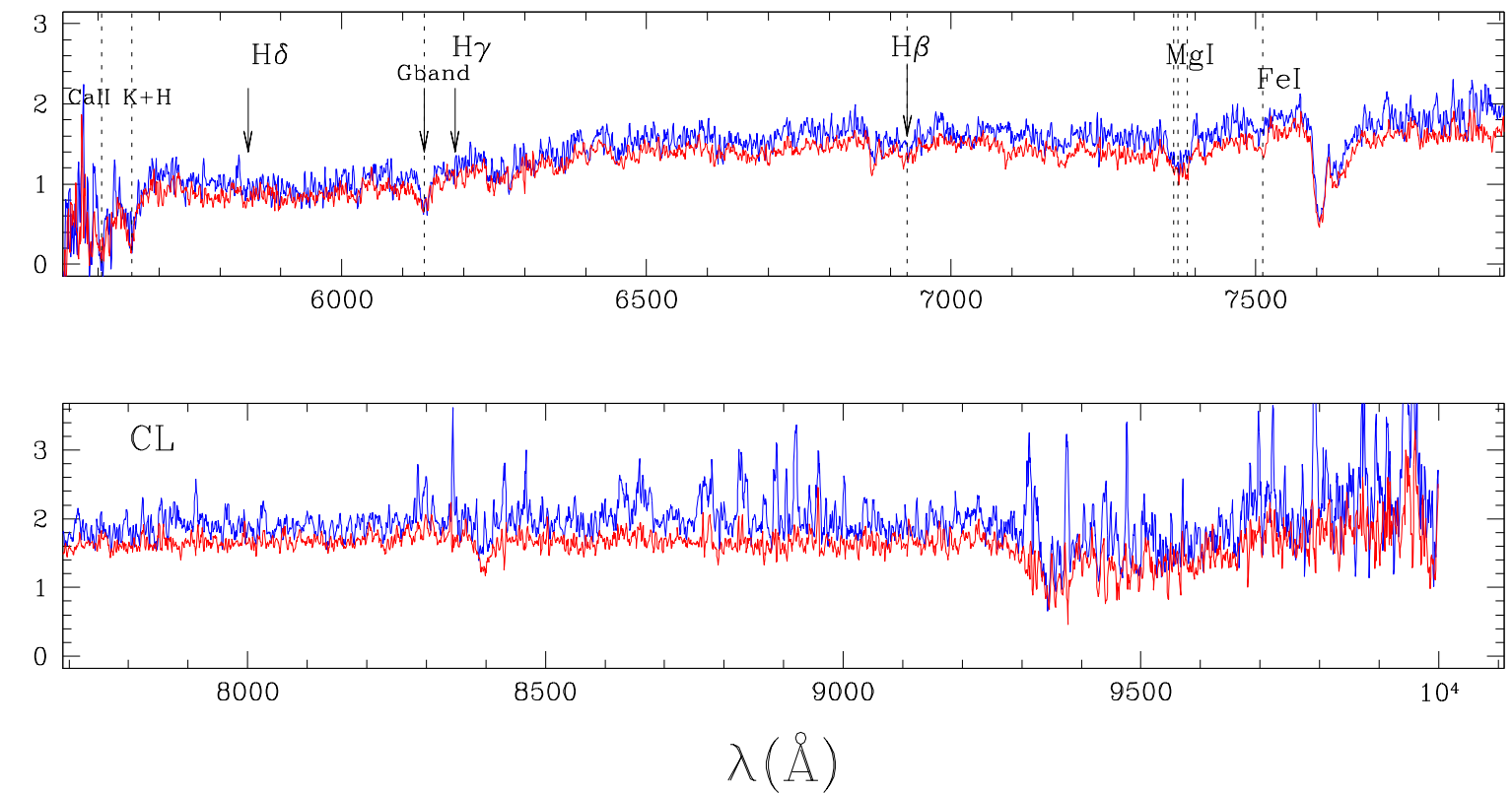

Figure A8. LBT/MODS spectra for CL, a candidate star cluster in DO 68, acquired with MODS1 (blue line) and MODS2 (red line). The system turned out to be a background early-type galaxy at z 0.42. 
Table B1. Observed emission fluxes for H in regions in DDO 68.

\begin{tabular}{|c|c|c|c|c|c|c|}
\hline Line & Reg-1 & Reg-3 & Reg-4 & Reg-7 & Reg-8 & Reg-9 \\
\hline [O II] $\lambda 3727$ & $6.20 \pm 0.09$ & $6.0 \pm 0.3$ & $4.8 \pm 0.1$ & $2.6 \pm 0.4$ & $11.2 \pm 0.6$ & $1.48 \pm 0.06$ \\
\hline H10 $\lambda 3978$ & $1.06 \pm 0.01$ & $0.39 \pm 0.05$ & - & $0.31 \pm 0.03$ & - & - \\
\hline He I $\lambda 3820$ & - & $0.27 \pm 0.01$ & - & - & - & - \\
\hline H9+He II $\lambda 3835$ & $1.44 \pm 0.06$ & $0.56 \pm 0.05$ & - & $0.3 \pm 0.1$ & - & - \\
\hline [Ne III] $\lambda 3869$ & $3.66 \pm 0.06$ & $1.18 \pm 0.05$ & $0.58 \pm 0.08$ & $0.38 \pm 0.01$ & - & - \\
\hline $\mathrm{H} 8+\mathrm{He}$ I $\lambda 3889$ & $4.4 \pm 0.2$ & $1.7 \pm 0.2$ & $0.76 \pm 0.04$ & $0.9 \pm 0.1$ & $0.72 \pm 0.08$ & - \\
\hline $\mathrm{H} \epsilon+\mathrm{He} \mathrm{I}+[\mathrm{Ne} \mathrm{III}] \lambda 3970$ & $4.51 \pm 0.08$ & $1.73 \pm 0.09$ & $0.67 \pm 0.06$ & $0.88 \pm 0.04$ & $0.64 \pm 0.07$ & - \\
\hline $\mathrm{H} \delta \lambda 4101$ & $6.1 \pm 0.1$ & $2.68 \pm 0.07$ & $1.28 \pm 0.01$ & $1.34 \pm 0.01$ & $1.1 \pm 0.1$ & $0.32 \pm 0.02$ \\
\hline $\mathrm{H} \gamma \lambda 4340$ & $11.5 \pm 0.4$ & $4.8 \pm 0.3$ & $2.61 \pm 0.03$ & $2.54 \pm 0.03$ & $2.01 \pm 0.03$ & $0.51 \pm 0.08$ \\
\hline [O III] $\lambda 4363$ & $1.42 \pm 0.04$ & $0.35 \pm 0.06$ & - & $0.094 \pm 0.005$ & - & - \\
\hline He I $\lambda 4471$ & $0.78 \pm 0.08$ & - & $0.15 \pm 0.01$ & $0.23 \pm 0.01$ & - & - \\
\hline He II (WR) $\lambda 4686$ & - & $0.9 \pm 0.2$ & - & - & - & - \\
\hline He II $\lambda 4686$ & $0.577 \pm 0.001$ & - & - & - & - & - \\
\hline $\mathrm{H} \beta \lambda 4861$ & $25.3 \pm 0.8$ & $10.9 \pm 0.6$ & $6.01 \pm 0.05$ & $5.65 \pm 0.07$ & $5.0 \pm 0.2$ & $1.06 \pm 0.01$ \\
\hline He I $\lambda 4922$ & $0.26 \pm 0.02$ & - & - & - & - & - \\
\hline [O III] $\lambda 4959$ & $16.1 \pm 0.5$ & $4.7 \pm 0.2$ & $1.57 \pm 0.05$ & $1.35 \pm 0.02$ & $1.03 \pm 0.05$ & $0.14 \pm 0.01$ \\
\hline [O III] $\lambda 5007$ & $46.5 \pm 1.5$ & $13.8 \pm 0.7$ & $4.5 \pm 0.1$ & $3.81 \pm 0.04$ & $3.2 \pm 0.2$ & $0.54 \pm 0.07$ \\
\hline He I $\lambda 5015$ & $0.59 \pm 0.03$ & - & - & - & - & - \\
\hline He I $\lambda 5876$ & $2.44 \pm 0.05$ & $1.12 \pm 0.01$ & $0.47 \pm 0.01$ & $0.56 \pm 0.01$ & $0.5 \pm 0.1$ & - \\
\hline [OI] $\lambda 6302$ & $0.135 \pm 0.009$ & - & - & $0.08 \pm 0.02$ & $0.39 \pm 0.02$ & - \\
\hline [S III] $\lambda 6312$ & $0.18 \pm 0.01$ & - & - & - & - & - \\
\hline $\mathrm{H} \alpha \lambda 6563$ & $77 \pm 2$ & $35.3 \pm 0.6$ & $18.5 \pm 0.1$ & $17.3 \pm 0.2$ & $17.2 \pm 0.1$ & $3.5 \pm 0.1$ \\
\hline [N II] $\lambda 6584$ & $0.29 \pm 0.04$ & $0.29 \pm 0.01$ & $0.18 \pm 0.02$ & $0.12 \pm 0.01$ & $0.51 \pm 0.02$ & - \\
\hline He I $\lambda 6678$ & $0.72 \pm 0.03$ & $0.324 \pm 0.005$ & $0.170 \pm 0.007$ & $0.18 \pm 0.01$ & $0.13 \pm 0.02$ & - \\
\hline [S II] $\lambda 6716$ & $0.59 \pm 0.01$ & $0.59 \pm 0.07$ & $0.5 \pm 0.2$ & $0.30 \pm 0.01$ & $1.58 \pm 0.01$ & $0.16 \pm 0.01$ \\
\hline [S II] $\lambda 6731$ & $0.40 \pm 0.02$ & $0.44 \pm 0.01$ & $0.20 \pm 0.05$ & $0.23 \pm 0.02$ & $1.14 \pm 0.05$ & $0.07 \pm 0.01$ \\
\hline He I $\lambda 7065$ & $0.645 \pm 0.001$ & $0.32 \pm 0.02$ & $0.16 \pm 0.01$ & $0.111 \pm 0.008$ & - & - \\
\hline [Ar III] $\lambda 7136$ & $0.49 \pm 0.01$ & $0.242 \pm 0.009$ & $0.102 \pm 0.006$ & $0.08 \pm 0.03$ & - & - \\
\hline He I $\lambda 7281$ & $0.118 \pm 0.006$ & - & - & - & - & - \\
\hline [O II] $\lambda 7320$ & $0.19 \pm 0.02$ & - & - & - & $0.23 \pm 0.02$ & - \\
\hline [O II] $\lambda 7330$ & $0.15 \pm 0.03$ & - & - & - & $0.27 \pm 0.04$ & - \\
\hline P10 $\lambda 9017$ & $0.484 \pm 0.003$ & - & - & - & - & - \\
\hline [S III] $\lambda 9069$ & $1.03 \pm 0.06$ & $0.45 \pm 0.03$ & $0.20 \pm 0.07$ & $0.16 \pm 0.05$ & $0.28 \pm 0.01$ & - \\
\hline P9 $\lambda 9229$ & $0.669 \pm 0.003$ & $0.34 \pm 0.05$ & - & - & - & - \\
\hline [S III] $\lambda 9532$ & $2.3 \pm 0.1$ & $1.1 \pm 0.2$ & - & - & - & - \\
\hline P8 $\lambda 9547$ & $0.95 \pm 0.04$ & - & - & - & - & - \\
\hline
\end{tabular}

The fluxes, in units of $10^{-16} \mathrm{ergs}^{-1} \mathrm{~cm}^{-2} \AA^{-1}$, are not corrected for reddening or for Balmer/Helium line absorption. 